\title{
Istituto
}

Nazionale

Sezione di ROMA

Piazzale Aldo Moro, 2

I-00185 Roma, Italy

Fisica

INFN-1335/02

Nucleare

May 2002

\section{Pair term in the Electromagnetic Current within the Front-Form Dynamics: Spin-0 Case}

\author{
J. P. B. C. de Melo ${ }^{a}$, T. Frederico ${ }^{b}$, E. Pace ${ }^{c}$ and G. Salmè ${ }^{d}$ \\ a Instituto de Fúsica Teórica, Universidade Estadual Paulista 0145-900, São Paulo, SP, \\ Brazil \\ ${ }^{b}$ Dep. de Física, Instituto Tecnológico da Aeronáutica, Centro Técnico Aeroespacial, \\ 12.228-900, São José dos Campos, São Paulo, Brazil \\ "Dipartimento di Fisica, Università di Roma "Tor Vergata" and Istituto Nazionale di Fisica \\ Nucleare, Sezione Tor Vergata, Via della Ricerca Scientifica 1, I-00133 Roma, Italy \\ d Istituto Nazionale di Fisica Nucleare, Sezione Roma I, P.le A. Moro 2, I-00185 Roma, \\ Italy
}

\begin{abstract}
The frame and scale dependence of the pair-term contribution to the electromagnetic form factor of a spin-zero composite system of two-fermions is studied within the Light Front. The form factor is evaluated from the plus-component of the current in the Breit frame, using for the first time a nonconstant, symmetric ansatz for the BetheSalpeter amplitude. The frame dependence is analyzed by allowing a nonvanishing plus component of the momentum transfer, while the dynamical scale is set by the masses of the constituents and by mass and size of the composite system. A transverse momentum distribution, associated with the Bethe-Salpeter amplitude, is introduced which allows to define strongly and weakly relativistic systems. In particular, for strongly relativistic systems, the pair term vanishes for the Drell-Yan condition, while is dominant for momentum transfer along the light-front direction. For a weakly relativistic system, fitted to the deuteron scale, the pair term is negligible up to momentum transfers of $1(\mathrm{GeV} / \mathrm{c})^{2}$. A comparison with results obtained within the Front-Form Hamiltonian dynamics with a fixed number of constituents is also presented.
\end{abstract}

To appear in Nucl. Phys. A 


\section{Introduction}

Present knowledge of the structure of hadrons and nuclei mainly comes from electroweak form factors, in elastic and transition regimes, and from deep-inelastic structure functions. To perform a meaningful comparison between theoretical models and the experimental data, one needs a description of the bound system of interacting constituents and a consistent current operator. Within a Hamiltonian approach, the state of the system is defined on a specified hypersurface of the space-time that does not contain timelike directions. Dirac identified three space-time hypersurfaces, adequate to define the state of a relativistic system, which correspond to different forms of relativistic Hamiltonian Dynamics, namely the Instant Form, the Front Form and the Point Form [i]].

In the Front-Form dynamics, the consistency between the current operator and the state of the hadron system has been discussed both from a field theoretical point of view and within approaches with a fixed number of particles. In field theory, the state has an infinite number of components in the Fock space [2]. However, for practical applications only the lowest Fock component, or valence component, is usually modeled and used in the calculations of electroweak form factors. In principle, the infinite set of coupled eigenvalue equations for the Hamiltonian operator in the Fock space can be replaced by an effective squared mass operator acting in the valence sector; at the same time, it is possible to express systematically the higher Fock-state components of the wave function as functionals of the lower ones [2, [3, 国. The effective electroweak current operator to be used with the valence component of the state can be consistently derived within the framework of the BetheSalpeter equation projected at equal light-front time, as recently shown in Refs. [5, 6]. (For recentinvestigation on other aspects of the Bethe-Salpeter equation within the light front, see, e.g. [7, 8]).

If a fixed number of interacting particles is assumed, then a Front-Form Hamiltonian dynamics (FFHD) can be developed. One can use the Bakamjian-Thomas construction [9], where an effective interaction in the mass operator can be chosen such that a unitary representation of the Poincaré group is possible. Taking the Front-Form spin operator as the free one and an interaction in the mass operator that commutes with such a spin operator, one is able to explicitly construct generators of the Poincaré group [10, 11]. In this case, the eigenfunctions of the mass operator are normalized to one, differently from the field theoretical valence component, which has in general a probability less than one 21]. A wide number of papers have been devoted to the evaluation of properties of hadrons and nuclei within the Front-Form Hamiltonian dynamics with a finite number of particles (see, e.g. Refs. [12, 13, 14, 15, 16, 17, 18], just to give a short account of some previous works). In particular, it should be noted that FFHD for the two-nucleon case yields the possibility to retain the large amount of successful phenomenology developed within a nonrelativistic approximation (see, e.g., [12, 19]). Usually, to obtain electroweak form factors of nuclear systems or hadrons, the Drell-Yan condition on the momentum transfer, i.e. $q^{+}=q^{0}+q^{3}=0$, and the matrix elements of the plus component of the current, $j^{+}$, are adopted. The physical argument often advocated in favour of these assumptions is that the production of pairs from the 
incoming photon (nonvalence or pair-term contribution) is suppressed in the Drell-Yan frame by light-front momentum conservation (see, e.g., [20]). This was indeed proved in schematic covariant field theoretical models, for spin-zero two-boson composite systems 21] and for the pion with pseudo-scalar coupling to the quarks 22]. However, for spin-one systems the pair term survives and contributes to $j^{+}$in the Drell-Yan frame [23, 24, 25]. Furthermore, in this case the pair term is necessary to keep the rotational invariance of the form factors for spin-one particles. Within FFHD, in the Drell-Yan frame, the matrix elements of the plus component of the current should fulfill the so called angular condition [26]. This constraint is not satisfied by a calculation considering only the Front-Form wave function and the impulse approximation. This approximation implies an ambiguity in extracting form factors for spinone systems, as shown for the deuteron 26, 12, 27] and for the rho-meson [28, 29, 30]. To solve this problem, related to the lacking of covariance for the impulse approximation current operator, some physically inspired combinations of matrix elements were proposed to extract form factors from the current, as the "good-component" approach 31] or the elimination of spurious contributions to the form factors 32.

To avoid the difficulties associated with these ambiguities, another approach was proposed in Ref. 33 and applied to the deuteron 19, 34, 35. In these works, it was shown that a current operator which satisfies the requirements of Poincaré, parity and time-reversal covariance, as well as hermiticity and current conservation, can be obtained from a one-body operator in the Breit frame with momentum transfer along the z-direction. Then, for any hadron system the electromagnetic form factors can be calculated from the wave function without ambiguities.

This major development in the calculation of the form factors from the Front-Form wave function is, however, confronted with the fact that, even in simple field-theoretical models, the pair production mechanism contributes in the $q^{+} \neq 0$ Breit frame, as was discussed in the calculation of the pion form factor from $j^{+}$in Ref. [36]. Therefore, within an approach with a fixed number of particles such a mechanism should be taken into account through an effective two-body current.

The aim of the present work is to investigate in a covariant model, based on a nonconstant, symmetric vertex function, the effect of the pair term in the evaluation of electromagnetic form factors for a pion-like system composed by two identical fermions. It is well known that, to avoid divergences in the evaluation of the covariant triangle-diagram for the form factor some regularization has to be introduced. In Refs. [22, 23, 24] a pion- $q \bar{q}$ vertex function, non symmetric in the four-momenta of the quarks, was adopted. However, a non symmetric vertex cannot be considered a realistic approximation of a $q \bar{q}$ bound-state amplitude and phenomenological problems arise (e. g., the form factor and the weak decay constant cannot be simultaneously reproduced) [22]. Another approach has been proposed in Ref. [36], where the fermion loop was regulated by considering a non-local photon vertex. In the present work, for describing the momentum part of the coupling between the constituents and the spin-0 system, we use a covariant model with the following form of the 
vertex function

$$
\Lambda(k, P)=\frac{C}{\left(k^{2}-m_{R}^{2}+\imath \epsilon\right)}+\frac{C}{\left((P-k)^{2}-m_{R}^{2}+\imath \epsilon\right)} .
$$

Differently from [22, 23, 24], we adopt a vertex function which is symmetric by the exchange of the momentum of the two fermions and implies a light-front valence wave function with the same property, as shown in the following Sections. In this way, we are simulating the symmetry properties of a Bethe-Salpeter amplitude derived from quantum field theory. The other main ingredient of our covariant model, for calculating the form factor of the composite system, is the electromagnetic current, that is taken in impulse approximation. It should be pointed out that within our approach such a current is conserved (see Sect. II). In the spirit of Ref. [36], we study the importance of the pair diagram evaluated in different Breit frames, which differ for the direction of the spatial part of the momentum transfer. We analyze and compare two systems that have relativistic or nonrelativistic nature, respectively. In order to better define this feature, we first construct the valence wave function from the symmetric ansatz for the Bethe-Salpeter amplitude and then, from this wave function, we build up the transverse-momentum distribution of the constituents. This momentum distribution plays a twofold role, allowing one: i) to make contact betwen our covariant model and dynamical models of the composite system, developed within approaches with a fixed number of particles, and, more important, ii) to quantitatively define the two limiting cases that we will consider, namely the strongly and weakly relativistic systems. In the first case, we make calculations for a spin-0 model, well suited for the pion. Then we compare these results with the ones obtained with a realistic pion wave function generated by a potential able to describe the meson spectroscopy [37]. In the second case, we artificially adjust the transverse momentum distribution of the model to the deuteron scale, to get insight into the pair-term contribution for a weakly relativistic system with a small average transverse momentum for the constituents (getting rid of the lengthy algebra of the $m_{1} \neq m_{2}$ case, corresponding to the actual case of charged heavy mesons).

The paper is organized as follows. In Sec. II, our model for the spin-zero, twofermion system with a symmetric vertex function is presented. In Sec. III, the light-front valence wave function for the covariant model is introduced, as well as the corresponding transverse momentum distribution and the corresponding elastic form factor. In Sec. IV, numerical results are presented for i) the electromagnetic form factor of our model, ii) the separate contribution of the pair term and iii) the form factor corresponding to the valence component. In Sec. V, we draw our conclusions.

\section{Electromagnetic form factor of a pion-like system}

In our model, the electromagnetic current for a two-fermion composite system with spin equal to 0 - i.e., a pion-like system considered as $q \bar{q}$ bound state - is calculated in oneloop approximation (triangle diagram), modelling the Bethe-Salpeter amplitude through a symmetric vertex function in momentum space with a pseudoscalar coupling between pion 
and quark degrees of freedom. This coupling is suggested by a simple effective Lagrangian (see, e.g. [38])

$$
\mathcal{L}_{I}=-\imath g \vec{\Phi} \cdot \bar{q} \gamma^{5} \vec{\tau} q
$$

The coupling constant $g$ is given by the Goldberg-Treiman relation at the quark level, namely $g=m / f_{\pi}$, with $m$ the mass of the constituents and $f_{\pi}$ the pion decay constant.

The electromagnetic current of $\pi^{+}$is obtained from the covariant expression corresponding to the triangle diagram (see, e.g., [39] and 40]):

$$
j^{\mu}=-\imath 2 e \frac{m^{2}}{f_{\pi}^{2}} N_{c} \int \frac{d^{4} k}{(2 \pi)^{4}} \operatorname{Tr}\left[S(k) \gamma^{5} S\left(k-P^{\prime}\right) \gamma^{\mu} S(k-P) \gamma^{5}\right] \Lambda\left(k, P^{\prime}\right) \Lambda(k, P),
$$

where $S(p)=\frac{1}{\not p-m+\imath \epsilon}, N_{c}=3$ is the number of colors, $P^{\mu}$ and $P^{\prime \mu}=P^{\mu}+q^{\mu}$ are the initial and final momenta of the system, $q^{\mu}$ is the momentum transfer and $k^{\mu}$ the spectator quark momentum. The factor 2 stems from isospin algebra. (Current conservation can be easily proven in the Breit frame: after performing the trace in $q \cdot j$, one notices that the integrand of the resulting expression is odd by changing $\vec{k} \rightarrow-\vec{k}$; this means that $q \cdot j$ is zero.)

In Eq. (3), we introduce the symmetric vertex function of Eq. (1). This vertex function produces a light-front wave function symmetric by the interchange of quark and antiquark momenta, and is not affected by the conceptual difficulties associated with the use of the nonsymmetric regulator, as mentioned in the Introduction (see Refs. 30, 36]). Since we have not specified the dynamics which drives the Bethe-Salpeter amplitude, we have to resort to a physical condition for normalizing the Bethe-Salpeter vertex. As a matter of fact, the normalization constant $C$ in the vertex function, Eq. (1), is fixed by imposing the charge normalization condition (i.e. the pion form factor at zero momentum transfer must be equal to 1$)$.

In our analysis we consider Breit frames, where the momentum transfer $q^{\mu}$ has the spatial component parallel to the $z-x$ plane. By using Front-Form variables, i.e. $k^{+}=$ $k^{0}+k^{3}, k^{-}=k^{0}-k^{3}, \vec{k}_{\perp} \equiv\left(k^{1}, k^{2}\right)$ one has

$$
\begin{aligned}
& q^{+}=-q^{-}=\sqrt{-q^{2}} \sin \alpha, \quad q_{x}=\sqrt{-q^{2}} \cos \alpha, \quad q_{y}=0 \\
& q^{2}=q^{+} q^{-}-\left(\vec{q}_{\perp}\right)^{2}
\end{aligned}
$$

The Drell-Yan condition $q^{+}=0$ is recovered with $\alpha=0$, while the $q^{+}=\sqrt{-q^{2}}$ condition [33 comes with $\alpha=90^{\circ}$. (Note that the angle $\theta$ of Ref. [36] corresponds to $\alpha+90^{\circ}$ ). The initial and final momenta of the composite spin-0 bound state are: $P^{0}=E=E^{\prime}=\sqrt{m_{B}^{2}-q^{2} / 4}$, $\vec{P}_{\perp}^{\prime}=-\vec{P}_{\perp}=\vec{q}_{\perp} / 2$ and $P_{z}^{\prime}=-P_{z}=q^{+} / 2$.

As well known, the pion form factor can be extracted from the covariant expression:

$$
j^{\mu}=e\left(P^{\mu}+P^{\prime \mu}\right) F_{\pi}\left(q^{2}\right) .
$$


If covariance and current conservation are fulfilled in a model calculation, one can obviously use any frame and any nonvanishing component of the current to calculate the electromagnetic form factor. We calculate the form factor for our pion model defined by Eq. (1) and Eq. (3), using the plus component of the current in the Breit frame with $\vec{q}$ in the $z-x$ plane. In the evaluation of the form factor one can single out two nonvanishing contributions in Eq. (3) [21, 22, 36, 41]:

$$
F_{\pi}\left(q^{2}\right)=F_{\pi}^{(I)}\left(q^{2}, \alpha\right)+F_{\pi}^{(I I)}\left(q^{2}, \alpha\right),
$$

where $F_{\pi}^{(I)}\left(q^{2}, \alpha\right)$ has the loop integration on $k^{+}$constrained by $0 \leq k^{+}<P^{+}$, see the light-front time-ordered diagram in Fig. 1(a), while $F_{\pi}^{(I I)}\left(q^{2}, \alpha\right)$ has the loop integration on $k^{+}$in the interval $P^{+} \leq k^{+} \leq P^{\prime+}$, see Fig. 1(b). The valence component of the pion contributes to $F_{\pi}^{(I)}\left(q^{2}, \alpha\right)$ only, but in our model it does not give the full result in $0 \leq k^{+}<P^{+}$, as discussed in detail in Sec. IV. The component $F_{\pi}^{(I I)}\left(q^{2}, \alpha\right)$ of the form factor is the contribution of the pair production mechanism from an incoming virtual photon with $q^{+}>0$.

The two contributions to the form factor obtained from $j^{+}$are given by the following expressions

$$
F_{\pi}^{(I)}\left(q^{2}, \alpha\right)=-\imath \frac{m^{2}}{\left(P^{+}+P^{\prime+}\right) f_{\pi}^{2}} \frac{N_{c}}{(2 \pi)^{4}} \int \frac{d^{2} k_{\perp} d k^{+} d k^{-} \theta\left(k^{+}\right) \theta\left(P^{+}-k^{+}\right)}{k^{+}\left(P^{+}-k^{+}\right)\left(P^{\prime+}-k^{+}\right)} \Pi\left(k, P, P^{\prime}\right)
$$

and

$$
F_{\pi}^{(I I)}\left(q^{2}, \alpha\right)=-\imath \frac{m^{2}}{\left(P^{+}+P^{\prime+}\right) f_{\pi}^{2}} \frac{N_{c}}{(2 \pi)^{4}} \int \frac{d^{2} k_{\perp} d k^{+} d k^{-} \theta\left(k^{+}-P^{+}\right) \theta\left(P^{\prime+}-k^{+}\right)}{k^{+}\left(P^{+}-k^{+}\right)\left(P^{\prime+}-k^{+}\right)} \Pi(k, P, P(\$)
$$

where

$$
\begin{aligned}
\Pi\left(k, P, P^{\prime}\right) & =\frac{\operatorname{Tr}\left[\mathcal{O}^{+}\right] \Lambda(k, P) \Lambda\left(k, P^{\prime}\right)}{\left(k^{-}-k_{\text {on }}^{-}+\imath \epsilon\right)\left(P^{-}-k^{-}-(P-k)_{\text {on }}^{-}+\frac{\imath \epsilon}{P^{+}-k^{+}}\right)} \\
& \times \frac{1}{\left(P^{\prime-}-k^{-}-\left(P^{\prime}-k\right)_{\text {on }}^{-}+\imath \epsilon\right)},
\end{aligned}
$$

with the on-energy-shell values of the individual momenta given by

$$
k_{\text {on }}^{-}=\frac{k_{\perp}^{2}+m^{2}}{k^{+}},(P-k)_{\text {on }}^{-}=\frac{(P-k)_{\perp}^{2}+m^{2}}{P^{+}-k^{+}} \text {and }\left(P^{\prime}-k\right)_{o n}^{-}=\frac{\left(P^{\prime}-k\right)_{\perp}^{2}+m^{2}}{P^{\prime+}-k^{+}} \text {. }
$$

In Eq. (9), the trace $\operatorname{Tr}\left[\mathcal{O}^{+}\right]$of the operator

$$
\mathcal{O}^{+}=(\not k+m) \gamma^{5}\left(\not k-\not P^{\prime}+m\right) \gamma^{+}(\not k-P+m) \gamma^{5},
$$

is given by:

$$
\begin{aligned}
\frac{1}{4} \operatorname{Tr}\left[\mathcal{O}^{+}\right] & =-k^{-}\left(P^{\prime+}-k^{+}\right)\left(P^{+}-k^{+}\right)+\left(k_{\perp}^{2}+m^{2}\right)\left(k^{+}-P^{+}-P^{\prime+}\right) \\
& -\frac{1}{2} \vec{k}_{\perp} \cdot\left(\vec{P}_{\perp}^{\prime}-\vec{P}_{\perp}\right)\left(P^{\prime+}-P^{+}\right)+\frac{1}{4} k^{+} q_{\perp}^{2} .
\end{aligned}
$$


The explicit form of the symmetric regulator function in Front-Form momentum coordinates is given by

$$
\begin{aligned}
\Lambda(k, P) & =C\left[k^{+}\left(k^{-}-\frac{k_{\perp}^{2}+m_{R}^{2}-\imath \epsilon}{k^{+}}\right)\right]^{-1} \\
& +C\left[\left(P^{+}-k^{+}\right)\left(P^{-}-k^{-}-\frac{(P-k)_{\perp}^{2}+m_{R}^{2}-\imath \epsilon}{P^{+}-k^{+}}\right)\right]^{-1}
\end{aligned}
$$

where the position of the poles for $k^{-}$clearly appears.

The detailed forms of $F_{\pi}^{(I)}$ and $F_{\pi}^{(I I)}$, after integrating over $k^{-}$, are given in the Appendices A and B, respectively. In what follows, we will discuss some general features of Eq. (7) and Eq. (8).

Since the integration range of $k^{+}$is $0 \leq k^{+}<P^{+}$in Eq. (7) and $P^{+} \leq k^{+} \leq P^{\prime+}$ in Eq. (8), then, the sign of the imaginary part of some of the poles in the $k^{-}$-complex plane changes (see Eq. (9) and Eq. (13)). The poles that have their imaginary part modified are

$$
k_{(1)}^{-}=P^{-}-(P-k)_{o n}^{-}+\frac{\imath \epsilon}{P^{+}-k^{+}}=P^{-}-\frac{(P-k)_{\perp}^{2}+m^{2}}{P^{+}-k^{+}}+\frac{\imath \epsilon}{P^{+}-k^{+}},
$$

and

$$
k_{(2)}^{-}=P^{-}-\frac{(P-k)_{\perp}^{2}+m_{R}^{2}}{P^{+}-k^{+}}+\frac{\imath \epsilon}{P^{+}-k^{+}} .
$$

The last one comes from the vertex function, Eq. (13). The difference in the sign of the imaginary parts of $k_{(1)}^{-}$and $k_{(2)}^{-}$for the intervals $0 \leq k^{+}<P^{+}$and $P^{+} \leq k^{+} \leq P^{\prime}$ is the mathematical signature of the pair production mechanism, which appears just in the second interval.

The sum of the contributions $F_{\pi}^{(I)}\left(q^{2}, \alpha\right)$ and $F_{\pi}^{(I I)}\left(q^{2}, \alpha\right)$ yields the covariant result, dependent upon $q^{2}$ only. Then the different directions of $\vec{q}$ in the Breit frame can only change the values of $F_{\pi}^{(I)}\left(q^{2}, \alpha\right)$ and $F_{\pi}^{(I I)}\left(q^{2}, \alpha\right)$, but not their sum. For instance, by chosing $q^{+}=0$ (i.e. $\alpha=0) F_{\pi}^{(I I)}\left(q^{2}, \alpha\right)$ vanishes and therefore $F_{\pi}^{(I)}\left(q^{2}, \alpha\right)$ alone gives the whole, covariant result [21].

It is interesting to note that in our model the pair term is linear in $q^{+}$, for small $q^{+}$, as one can verify by direct inspection of the structure of Eq. (8), once the $k^{-}$integration is performed. As a matter of fact, the contour for the Cauchy integration in the calculation of the pair diagram can be closed in the upper complex $k^{-}$semi-plane (see Appendix B), and consequently the poles in the integrand of Eq. (\$) are

$$
k_{(3)}^{-}=P^{\prime-}-\left(P^{\prime}-k\right)_{o n}^{-}+\imath \epsilon=P^{\prime-}-\frac{\left(P^{\prime}-k\right)_{\perp}^{2}+m^{2}}{P^{\prime+}-k^{+}}+\imath \epsilon,
$$

and

$$
k_{(4)}^{-}=P^{\prime-}-\frac{\left(P^{\prime}-k\right)_{\perp}^{2}+m_{R}^{2}}{P^{\prime+}-k^{+}}+\imath \epsilon .
$$


The first pole, Eq. (16), comes from the last factor of Eq. (9), while the second pole, Eq. (17) comes from $\Lambda\left(P^{\prime}, k\right)$. The position of both poles in $k^{-}$are $\sim\left(q^{+}\right)^{-1}$ in the limit of $q^{+} \rightarrow 0$. Then, in order to find the dependence of $F_{\pi}^{(I I)}\left(q^{2}, \alpha\right)$ on $q^{+}$in this limit, it is enough to count the power of $q^{+}$in Eq. (8), when the residues are evaluated. The phasespace factor in the denominator of Eq. (8) is of the order of $\left(q^{+}\right)^{2}$. Then, let us consider the trace, Eq. (12), with the proper values of $k^{-}$(see Appendix B). The first term and third one are $\sim q^{+}$, the second term and the fourth one are of order $\left(q^{+}\right)^{0}$. Then the trace is of order $\left(q^{+}\right)^{0}$. Evaluating the contribution to the residues from the remaining part of Eq. (9), we found that $\Pi\left(k, P, P^{\prime}\right)$ is of the order of $\left(q^{+}\right)^{2}$. Therefore the integrand goes to a constant for $q^{+} \rightarrow 0$, and thus $F_{\pi}^{(I I)}\left(q^{2}, \alpha\right)$ is proportional to $q^{+}$in this limit, because of the range of the $k^{+}$integration.

A relevant feature in the analysis of the form factor is given by the presence of a contribution which is instantaneous in the light-front time, and is produced by the instantaneous term present in the Dirac propagator. As a matter of fact, the Dirac propagator can be decomposed using the Front-Form momenta as follows [2]

$$
\frac{\not k+m}{k^{2}-m^{2}+\imath \epsilon}=\frac{\not k_{o n}+m}{k^{+}\left(k^{-}-k_{o n}^{-}+\frac{\imath \epsilon}{k^{+}}\right)}+\frac{\gamma^{+}}{2 k^{+}},
$$

where the second term, proportional to $\gamma^{+}$, is an instantaneous term in the light-front time. It should be pointed out that the instantaneous contribution to the form factor is produced only by the spectator fermion. Indeed, the instantaneous terms pertaining to the other propagators do not contribute, because of the factor $\gamma^{+}$from the current and the property $\left(\gamma^{+}\right)^{2}=0$.

In our symmetric model, the instantaneous term of Eq. (18) contributes both to $F_{\pi}^{(I)}\left(q^{2}, \alpha\right)$ and $F_{\pi}^{(I I)}\left(q^{2}, \alpha\right)$, due to the analytic structure of the symmetric vertex function of Eq. (11). These contributions are of nonvalence nature, since they cannot be reduced to the impulse approximation with the valence wave function.

\section{Valence Light-front wave function}

The valence component of the light-front wave function can be obtained from the BetheSalpeter amplitude eliminating the relative light-front time, i.e., constraining to equal lightfront time the external space-time coordinates of the two fermions, after dropping the instantaneous terms of the external Dirac propagators [6]. Physically, in the external legs only light-front propagating particles are allowed. It is worth noting that the effect of the instantaneous terms, which are present in a Bethe-Salpeter approach, could be included in effective (many-body) operators to be used together with the valence wave function 6].

In the present model the Bethe-Salpeter amplitude is

$$
\Psi(k, P)=\frac{m}{f_{\pi}} \frac{\not k+m}{k^{2}-m^{2}+\imath \epsilon} \gamma^{5} \Lambda(k, P) \frac{\not k-P+m}{(k-P)^{2}-m^{2}+\imath \epsilon} .
$$


The momentum part of the valence component of the light-front wave function, $\Phi\left(k^{+}, \vec{k}_{\perp} ; P^{+}, \vec{P}_{\perp}\right)$, can be obtained by eliminating out from Eq. (19): i) the instantaneous terms, ii) the factors containing gamma matrices in the numerator, and iii) the $k^{+}$and $\left(P^{+}-k^{+}\right)$factors appearing in the denominator. Then, after introducing the explicit expression for $\Lambda$, one has to integrate over $k^{-}$, viz.

$$
\begin{aligned}
\Phi\left(k^{+}, \vec{k}_{\perp} ; P^{+}, \vec{P}_{\perp}\right) & =\imath \mathcal{N} \int \frac{d k^{-}}{2 \pi} \frac{1}{\left(k^{-}-k_{o n}^{-}+\frac{\imath \epsilon}{k^{+}}\right)\left(P^{-}-k^{-}-(P-k)_{o n}^{-}+\frac{\imath \epsilon}{P^{+}-k^{+}}\right)} \\
& \times\left(\frac{1}{k^{2}-m_{R}^{2}+\imath \epsilon}+\frac{1}{(P-k)^{2}-m_{R}^{2}+\imath \epsilon}\right)
\end{aligned}
$$

where $\mathcal{N}$ is a normalization factor

$$
\mathcal{N}=\sqrt{N_{c}} C \frac{m}{f_{\pi}}
$$

Performing the $k^{-}$integration in Eq. (20), one has

$$
\begin{aligned}
\Phi\left(k^{+}, \vec{k}_{\perp} ; P^{+}, \vec{P}_{\perp}\right)=\frac{P^{+}}{m_{\pi}^{2}-M_{0}^{2}} & {\left[\frac{\mathcal{N}}{(1-x)\left(m_{\pi}^{2}-\mathcal{M}^{2}\left(m^{2}, m_{R}^{2}\right)\right)}\right.} \\
& \left.+\frac{\mathcal{N}}{x\left(m_{\pi}^{2}-\mathcal{M}^{2}\left(m_{R}^{2}, m^{2}\right)\right)}\right],
\end{aligned}
$$

where $x=k^{+} / P^{+}$, with $0 \leq x \leq 1 ; \mathcal{M}^{2}\left(m_{a}^{2}, m_{b}^{2}\right)=\frac{k_{\perp}^{2}+m_{a}^{2}}{x}+\frac{(P-k)_{\perp}^{2}+m_{b}^{2}}{1-x}-P_{\perp}^{2}$; and the square of the free mass is $M_{0}^{2}=\mathcal{M}^{2}\left(m^{2}, m^{2}\right)$. Since we have chosen a nonconstant, symmetric $\Lambda$, a second term appears in Eq. (22), differently from Ref. [22], and then the momentum part of the wave function becomes symmetric by the exchange of the momenta of the two constituents.

By using only the valence component and generalizing the results of Refs. [22, 38], the electromagnetic form factor, $F_{\pi}^{(W F)}$, evaluated in the Breit frame is written as follows

$$
\begin{aligned}
F_{\pi}^{(W F)}\left(q^{2}, \alpha\right)=\frac{1}{2 \pi^{3}\left(P^{\prime+}+P^{+}\right)} & \quad \int \frac{d^{2} k_{\perp} d k^{+} \theta\left(k^{+}\right) \theta\left(P^{+}-k^{+}\right)}{k^{+}\left(P^{+}-k^{+}\right)\left(P^{\prime+}-k^{+}\right)} \Phi\left(k^{+}, \vec{k}_{\perp} ; P^{\prime+}, \frac{\vec{q}_{\perp}}{2}\right) \\
\times & {\left[k_{o n}^{-} P^{+} P^{\prime+}+\frac{1}{2} \vec{k}_{\perp} \cdot \vec{q}_{\perp}\left(P^{\prime+}-P^{+}\right)-\frac{1}{4} k^{+} q_{\perp}^{2}\right] } \\
\times & \times\left(k^{+}, \vec{k}_{\perp} ; P^{+},-\frac{\vec{q}_{\perp}}{2}\right),
\end{aligned}
$$

with $k_{\text {on }}^{-}=\left(k_{\perp}^{2}+m^{2}\right) / k^{+}$(see Eq. (10)). Once the normalization constant $C$ is determined from the condition $F_{\pi}(0)=1$ in Eq. (6), the value of $F_{\pi}^{(W F)}$ for $q^{2}=0$ yields the probability (independent of $\alpha$ ) of the valence $q \bar{q}$ component in the pion, $\eta$. It should be pointed out that a value of $\eta<1$ is expected, if the non-valence contributions are important, and this is just what occurs in our model (see Sect. IV for details), differently from the case of a nonsymmetric ansatz for the Bethe-Salpeter amplitude, where $\eta=1$ [22]. 
Equation (23) represents a point of contact between a field theoretical approach and the FFHD with a fixed number of particles, adopting an impulse approximation current operator. Indeed, including a proper factor in $\Phi$, namely $\sqrt{M_{0} / P^{+}}$and normalizing the wave function one can recover the FFHD expression for the form factor, $F_{\pi}^{F F H D}$. In particular, putting $\alpha=0^{\circ}$, one obtains the standard FFHD expression in the frame $q^{+}=0$ [13].

The valence component of the light-front wave function, Eq. (20), is not an eigenfunction of the total angular momentum, since it is only one of the components of the pion state in the Fock space. Therefore, we cannot directly compare the valence wave function of the present model with the wave functions corresponding to approaches with a fixed number of particles [17], which are eigenfunctions of the intrinsic angular momentum [10]. However, in order to make contact with dynamical models, we introduce the transverse momentum probability density

$$
f\left(k_{\perp}\right)=\frac{1}{4 \pi^{3} m_{\pi}} \int_{0}^{2 \pi} d \phi \int_{0}^{m_{\pi}} \frac{d k^{+} M_{0}^{2}}{k^{+}\left(m_{\pi}-k^{+}\right)} \Phi^{2}\left(k^{+}, \vec{k}_{\perp} ; m_{\pi}, \overrightarrow{0}\right)
$$

By integrating $f\left(k_{\perp}\right)$ over $\vec{k}_{\perp}$, we obtain the probability of the valence component in the pion:

$$
\eta=\int_{0}^{\infty} d k_{\perp} k_{\perp} f\left(k_{\perp}\right)
$$

Furthermore, the transverse momentum probability density vs $k_{\perp} / m$ results to be very useful for a quantitative definition of strongly and weakly relativistic composite systems. Indeed high values of the transverse momentum distribution for $k_{\perp} / m>1$ are a distinctive feature of a strongly relativistic system. In particular, in Sect. IV $f\left(k_{\perp}\right)$ will allow us to investigate the influence of the dynamical scale of the system on the role played by the pair term.

Another relevant quantity to be used for constraining the parameters of our model is the pion decay constant, $f_{\pi}$. It is defined through the matrix element of the partially conserved axial-vector current

$$
P_{\mu}<0\left|A_{i}^{\mu}\right| \pi_{j}>=\imath m_{\pi}^{2} f_{\pi} \delta_{i j} .
$$

Following Ref. 38], we take $A_{i}^{\mu}=\bar{q} \gamma^{\mu} \gamma^{5} \frac{\tau_{i}}{2} q$ and adopt our ansatz for the pion- $\bar{q} q$ vertex function. In this way we obtain

$$
\imath P^{2} f_{\pi}=\frac{m}{f_{\pi}} N_{c} \int \frac{d^{4} k}{(2 \pi)^{4}} \operatorname{Tr}\left[P \gamma^{5} S(k) \gamma^{5} S(k-P)\right] \Lambda(k, P),
$$

and integrating on $k^{-}$, one gets $f_{\pi}$ in terms of the valence component of our model:

$$
f_{\pi}=\frac{m \sqrt{N_{c}}}{4 \pi^{3}} \int \frac{d^{2} k_{\perp} d k^{+}}{k^{+}\left(m_{\pi}-k^{+}\right)} \Phi\left(k^{+}, \vec{k}_{\perp} ; m_{\pi}, \overrightarrow{0}\right)
$$




\section{$4 \quad$ Numerical Results}

\subsection{Pion model}

In our model, we have two free parameters for the pion: the constituent quark mass, $m$, and the regulator mass, $m_{R}$. The constituent quark mass is chosen as $m=0.220 \mathrm{GeV}$, adequate for the meson phenomenology [37, 38, 42]. The regulator mass $m_{R}=0.6 \mathrm{GeV}$ is found by fitting Eq. (28) to the experimental value $f_{\pi}^{e x p}=92.4 \mathrm{MeV}$. For the pion mass we use the experimental value of $0.140 \mathrm{GeV}$. As a consequence, the charge radius, obtained from $\left\langle r^{2}\right\rangle=6 \frac{\partial}{\partial q^{2}} F_{\pi}$, comes out to be $0.74 \mathrm{fm}$, which is about $10 \%$ larger than the experimental value $\left(r_{\text {exp }}=0.67 \pm 0.02 \mathrm{fm}\right.$ [43]).

In Fig. 2, the results for the pion form factor are shown and compared to the data of Refs. 44, 45, 46, 47, 48. The full-model calculations, Eq. (6), nicely agree with the new data for the pion form factor [44. Therefore, our model, based on a non-constant, symmetric vertex can reproduce the form factor data consistently with the experimental value of $f_{\pi}$, while for the nonsymmetric regulator this was not possible[22]. Remarkably, in order to reproduce simultaneously $f_{\pi}$ and the experimental form factor within our model, the constituent quark mass has to be chosen in the range between 0.2 and $0.3 \mathrm{GeV}$. Compatibility between form factor and decay constant has been also achieved in [36], where a constant pion- $q \bar{q}$ vertex and a nonlocal photon vertex were adopted.

The form factor in the Breit frame with $\alpha=0^{\circ}\left(q^{+}=0\right)$, where the pair-term contribution is zero, is identical to the sum of $F_{\pi}^{(I)}$ and $F_{\pi}^{(I I)}$, calculated in the frame corresponding to $\alpha=90^{\circ}$, as it must be for a covariant model. In Fig. $2, F_{\pi}^{(I)}$ and $F_{\pi}^{(I I)}$, for $\alpha=90^{\circ}$, are also shown. Differently from the case $\alpha=0^{\circ}$, for $\alpha=90^{\circ}$ the form factor is dominated by the pair production process, except near $q^{2}=0$. At high values of the momentum transfer the form factor is completely exhausted by the pair-term contribution. It is worth noting that such a dominance is mainly due to a kinematical effect and appears to be fairly model independent (see also Ref.[36]). A qualitative argument, which is applicable to the reference frame with $q^{+}=\sqrt{-q^{2}}$ and essentially follows the work of Sawicki[21], is given in what follows.

Since the form factor is dimensionless, one can write qualitatively

$$
F_{\pi}^{(I)}\left(q^{2}, \alpha\right) \sim \int_{0}^{P^{+}} \frac{d k^{+}}{P^{+}+P^{\prime+}} \sim \frac{P^{+}}{P^{\prime+}+P^{+}} .
$$

At the same time, see the discussion in Sect. II, the contribution of the pair production amplitude to the form factor, is roughly

$$
F_{\pi}^{(I I)}\left(q^{2}, \alpha\right) \sim \int_{P^{+}}^{P^{+}} \frac{d k^{+}}{P^{+}+P^{\prime+}} \sim \frac{q^{+}}{P^{\prime+}+P^{+}} .
$$

The validity of such an approximation is related to the momentum cutoff in the vertex function, and therefore it is not reliable for large values of $q^{+}$. Recalling that for $\alpha=90^{\circ}$

$$
P^{+}=\sqrt{m_{\pi}^{2}+\left(\frac{q^{+}}{2}\right)^{2}}-\frac{q^{+}}{2}
$$


the estimate of the ratio of the above contributions is given by

$$
\frac{F_{\pi}^{(I)}\left(q^{2}, \alpha=90^{\circ}\right)}{F_{\pi}^{(I I)}\left(q^{2}, \alpha=90^{\circ}\right)} \sim \sqrt{\left(\frac{m_{\pi}}{q^{+}}\right)^{2}+\frac{1}{4}}-\frac{1}{2} .
$$

At the qualitative level, we can roughly say from Eq. (32) that the two contributions to the form factor are expected to have about the same magnitude when $-q^{2}=-q_{(I / I I)}^{2}=$ $m_{\pi}^{2} / 2$, which gives for the pion $-q_{(I / I I)}^{2}=0.01(\mathrm{GeV} / \mathrm{c})^{2}$. Our model calculation yields $-q_{(I / I I)}^{2}=0.03(\mathrm{GeV} / \mathrm{c})^{2}$. In Ref. [36], for the kaon it was shown that the pair term contribution becomes dominant for $-q_{(I / I I)}^{2} \approx 0.2(\mathrm{GeV} / \mathrm{c})^{2}$, while our estimate (Eq. (32)) gives $-q_{(I / I I)}^{2}=m_{K}^{2} / 2=0.13(\mathrm{GeV} / \mathrm{c})^{2}$. Therefore, one could argue that our crude estimate is able to give a momentum scale where sizeable effects due to the pair production term are expected for $\alpha=90^{\circ}$.

The contributions of the instantaneous part of the Dirac propagator to $F_{\pi}^{(I)}\left(q^{2}, \alpha\right)$ and $F_{\pi}^{(I I)}\left(q^{2}, \alpha\right)$, called $F_{\pi \text { inst }}^{(I)}\left(q^{2}, \alpha\right)$ and $F_{\pi \text { inst }}^{(I I)}\left(q^{2}, \alpha\right)$, respectively, are also shown in Fig. 2 for $\alpha=90^{\circ}$. One can physically understand why in $F_{\pi}^{(I I)}\left(q^{2}, \alpha\right)$ the instantaneous part is important and dominates at high momentum transfers. The interpretation is the following: in principle, see Fig. 1 (b), the spectator quark can be exchanged between incoming and outgoing pion at a given instant $x^{+}$, while the pair of quark and antiquark has been produced by the virtual photon at an earlier stage. As the magnitude of the momentum $q^{-}\left(=-q^{+}\right)$ increases, the time fluctuation for the virtual process decreases and favours the instantaneous exchange of the spectator quark between the initial and final pion. In fact, Fig. 2 shows the dominance of $F_{\pi}^{(I I)}$ inst $\left(q^{2}, \alpha\right)$ in the pion form factor as the momentum transfer increases. It is worth noting that the value of $F_{\pi \text { inst }}^{(I)}\left(q^{2}, \alpha\right)$ is nonzero because of the specific analytic structure of the vertex. As a matter of fact, $F_{\pi}^{(I)}$ inst $\left(q^{2}, \alpha\right)$ is nonzero, because of the presence of a pole in Eq. (7) at $k^{-}=\left(k_{\perp}^{2}+m_{R}^{2}\right) / k^{+}$in the vertex function $\Lambda$, Eq. (13), when one choses to close the contour for the Cauchy integration in the lower complex semi-plane of $k^{-}$.

In Fig. 3, the results for the various contributions to the pion form factor for $-q^{2}=1$ $(\mathrm{GeV} / \mathrm{c})^{2}$ as a function of the angle $\alpha$ are shown. For increasing angles, the form factor changes smoothly from valence to pair-term or nonvalence dominance.

In Fig. 4, we compare the results for $F_{\pi}^{(I)}$, Eq. (7), and $F_{\pi}^{(W F)}$, Eq. (23), where the light-front valence component of the model, defined according to Eq. (22) is used. The absolute normalization of the valence component $\eta$, i.e., the probability of the $q \bar{q}$ Fock-state component in the pion, is calculated to be $\eta=0.77$, differently from the nonsymmetric regulator model of Ref. [22], where $\eta=1$. The symmetric form of the vertex implies contributions from many poles to the form factor and the presence of many poles makes the valence component comparatively smaller than in the nonsymmetric case. In a previous work on DIS, based on a wave function contribution only, [38] a renormalization around $0.5-0.75$ was necessary to fit the data. To compare $F_{\pi}^{(I)}$ and $F_{\pi}^{(W F)}$, we have arbitrarily normalized $F_{\pi}^{(W F)}(0)$ to 1 . As shown in Fig. 4, the momentum behaviour of $F_{\pi}^{(I)}$ and $F_{\pi}^{(W F)}$ is almost the same, independently of the reference frame, for $\alpha$ between $0^{\circ}$ and $90^{\circ}$. This means that 
our kinematical argument, about the suppression of $F_{\pi}^{(I)}\left(q^{2}, \alpha\right)$ with respect to the full form factor in the $q^{+}=\sqrt{-q^{2}}$ frame, could be extended to $F_{\pi}^{(W F)}\left(q^{2}, \alpha\right)$ as well.

Once $F_{\pi}^{(W F)}(0)$ is arbitrarily normalized to 1 , namely $C \rightarrow C / \sqrt{\eta}$ in Eq. (21), a good description of the form factor data for $\alpha=0^{\circ}$ is achieved, as one can deduce from Fig. 4, but

$f_{\pi}^{(W F)}=105 \mathrm{MeV}$, which overestimates the experimental value, since $f_{\pi}^{(W F)}=f_{\pi} / \sqrt{\eta}$. The value of $f_{\pi}^{(W F)}$ is similar to the one found for nonsymmetric models, once the form factor below $2(\mathrm{GeV} / \mathrm{c})^{2}$ is fitted (see e. g. [22]).

As discussed in Sect. III, $F_{\pi}^{(W F)}$ can be formally related to the form factor obtained within FFHD, where only the valence contribution appears. Since the momentum behaviour of $F_{\pi}^{(I)}$ and $F_{\pi}^{(W F)}$ is almost the same, then one can argue that $F_{\pi}^{F F H D}$ should correspond to the contribution of $F_{\pi}^{(I)}$. This means that $F_{\pi}^{F F H D}$ calculated in the Drell-Yan frame could represent a good effective approach for evaluating the form factor of the pion. Let us note that in FFHD the pair term can appear only as a contribution from two-body currents.

\subsection{Weakly relativistic systems}

In order to investigate the sensitivity of the pair term upon the dynamical scale of the composite system, we consider a case sharply different from the pion one. We adjust our model to the deuteron scale, and use for the mass of the system the value $m_{D}=1.874$ $\mathrm{GeV}$ and for the mass of the constituents $m=0.938 \mathrm{GeV}$. We adopt a regulator mass of $m_{R}=1.1 \mathrm{GeV}$ that gives a mean square radius of $3.25 \mathrm{fm}^{2}$ comparable to the difference, $r_{D, \text { exp }}^{2}-r_{p, \text { exp }}^{2}$, between the experimental values of the deuteron and proton mean square radii. In Fig. 5, we show the results of the form factor calculations for our mock deuteron. According to our qualitative kinematical estimate given in the previous subsection (see Eq. (32), with $m_{D}$ replacing $\left.m_{\pi}\right)$, at $-q^{2} \sim 2(\mathrm{GeV} / \mathrm{c})^{2}$ the pair term in the Breit frame with $q^{+}=\sqrt{-q^{2}}$ is expected to be as large as $F^{(I)}$. As a matter of fact, at this momentum transfer, the pair term is only about $15 \%$ of the form factor, as shown in Fig. 5. Indeed, the kinematical estimate does not work for a system with a rapidly decreasing momentum distribution. Moreover, we observe that the instantaneous term of the Dirac propagator gives a small contribution to the pair term, as well as to $F^{(I)}\left(q^{2}, \alpha=90^{\circ}\right)$. This fact is related to the basically nonrelativistic nature of the costituents in our mock deuteron. As illustrated in Fig. 6, the dimensionless product of $k_{\perp}^{2}$ times the transverse probability density, Eq. (24), is peaked at $k_{\perp}=0.06 \mathrm{~m}$ for our model. For the sake of comparison, in Fig. 6 it is also shown the transverse probability density for an actual deuteron; in particular the density has been calculated from the deuteron wave function corresponding to the Reid soft-core model of the deuteron 49. Remarkably, the same overall behaviour has been obtained in both cases. On the contrary the pion is a strongly relativistic system. For our pion model $k_{\perp}^{2} f\left(k_{\perp}\right)$ is peaked at $k_{\perp} \simeq m$, while for the FFHD model of Refs. [16, 17] the peak occurs around $k_{\perp}=2.5 \mathrm{~m}$. The FFHD model of Refs. 16, 17] is based on a mass operator with the effective interaction of Ref.[37], which includes one-gluon-exchange and linear confinement terms. The pion model from this mass operator, in the $q^{+}=0$ frame, has a charge radius of $0.46 \mathrm{fm}$ for pointlike constituent quarks. This value largely explains why the position of 
the peak appears at an higher value of the transverse momentum with respect to our model, which has a radius of $0.74 \mathrm{fm}$.

In summary, for a weakly relativistic system with spin equal to 0 , the valence contribution to the form factor yields a good approximation of the whole form factor up to $-q^{2}=2(\mathrm{GeV} / \mathrm{c})^{2}$ in any reference frame. Therefore, one can argue that the evaluation of $F^{F F H D}$ can be considered a reliable approximation to the full form factor in this case.

\subsection{Pair-term contribution and FFHD estimate}

In order to study in more detail the pair-term contribution, we show in Fig. 7 the ratio between $F^{(I I)}\left(q^{2}, \alpha=90^{\circ}\right)$ and the full form factor. We compare the results of our covariant model

$$
R^{(C O V)}=\frac{F^{(I I)}\left(q^{2}, \alpha=90^{\circ}\right)}{F\left(q^{2}\right)}
$$

with the kinematical estimate, Eqs. (29) and (30), given by

$$
R^{(K I N)}=\frac{q^{+}}{q^{+}+P^{+}}
$$

This kinematical estimate of the pair term works fairly well for the pion, while it overestimates the contribution of $F^{(I I)}$ for the weakly relativistic system, due to the rapid fall-off of the momentum distribution which makes Eq. (30) unreliable. In Fig. 7, we also report the following ratio

$$
R^{(F F H D)}=\frac{F_{\pi}^{(D Y)}\left(q^{2}\right)-F_{\pi}^{(L P S)}\left(q^{2}\right)}{F_{\pi}^{(D Y)}\left(q^{2}\right)} .
$$

where $F_{\pi}^{(D Y)}\left(q^{2}\right)$ and $F_{\pi}^{(L P S)}\left(q^{2}\right)$ are the pion form factor evaluated in the Drell-Yan frame and in the $q^{+}=\sqrt{-q^{2}}$ frame, respectively, within the FFHD model of Ref. [17], using pointlike constituent quarks and the one-body current. Such a ratio could yield insight on the relevance in FFHD of two-body current contributions corresponding to the pair term. Indeed, $F_{\pi}^{(D Y)}\left(q^{2}\right)$ represents an estimate of $F_{\pi}^{(I)}\left(q^{2}, \alpha=0^{o}\right)=F_{\pi}\left(q^{2}\right)$, while $F_{\pi}^{(L P S)}\left(q^{2}\right)$ should describe $F_{\pi}^{(I)}\left(q^{2}, \alpha=90^{\circ}\right.$ ) (cf. comments to Fig. 4) and therefore one can argue that $R^{(F F H D)} \sim R^{(C O V)} \sim R^{(K I N)}$. As a matter of fact, the ratio $R^{(F F H D)}$ qualitatively agrees with the results of our covariant model and with the model-independent kinematical estimate (see Fig. 7). Thus, the suppression of $F_{\pi}^{F F H D}$ in the $q^{+}=\sqrt{-q^{2}}$ frame with respect to the Drell-Yan frame can be explained in terms of a missing contribution in the current, that we could identify with two-body current contributions related to the pair term.

For the sake of completeness, $R^{(C O V)}$ and $R^{(K I N)}$ are also shown in Fig. 7 for a weakly relativistic sytem. Since $R^{(K I N)}>>R^{(C O V)}$, one can rely only on model calculations for inferring the dominance of the valence contribution with respect to the nonvalence, over a wide range of momentum transfer. We have also evaluated $R^{(F F H D)}$ for our mock deuteron and we have found $R^{(F F H D)} \sim R^{(C O V)}$. This result confirms the small effect of the pair term for a spin-0 weakly relativistic system. 


\section{Conclusion}

Within a covariant model with a vertex function which is symmetric in the momentum space of the constituents, we have calculated the electromagnetic form factor for a two-fermion, pion-like system. Such a model has allowed us to perform a detailed analysis of the pairterm contribution both changing the orientation of the Breit frame, where the form factor is calculated, and the dynamical scale of the system.

We have used a pseudoscalar coupling for the pion- $q \bar{q}$ vertex and a current for the point-like constituents in impulse approximation. It is worth noting that in our approach the current of the whole system is conserved. We have adopted for the first time a nonconstant, symmetric ansatz for the Bethe-Salpeter amplitude, and we have obtained the valence component of the pion state by projecting out the Bethe-Salpeter amplitude on the $x^{+}=0$ hypersurface. The symmetric vertex function implies a valence wave function with the same property. In this way we have overcomed some previous conceptual and phenomenological difficulties related to the use of a nonsymmetric regulator for describing the pion. As a matter of fact, the form factor and the weak decay constant cannot be simultaneously reproduced with a nonsymmetric vertex function (see, e. g., [22]).

In our covariant model for the pion, the two free parameters, the constituent quark mass $(m)$ and the regulator mass $\left(m_{R}\right)$, have been fixed as follows: i) the constituent quark mass was chosen as $m=0.220 \mathrm{GeV}$ from the meson spectroscopy [37], ii) the regulator mass $m_{R}=0.6 \mathrm{GeV}$ was found by fitting the experimental weak decay constant, $f_{\pi}^{e x p}=92.4 \mathrm{MeV}$. As a consequence, the form factor obtained in our covariant model nicely agrees with the new data for the pion form factor [44]. It should be pointed out that, if we wish to reproduce simultaneously $f_{\pi}$ and the form factor data with our approach, the constituent quark mass should be chosen in the range between 0.2 and $0.3 \mathrm{GeV}$. Another interesting outcome of our symmetric model is that the probability of the pion valence component, $\eta$, is less than 1 (about 75\%), at variance with previous covariant calculations [22, 36] where $\eta=1$. The em form factor of the pion-like system has been decomposed in two contributions, with the second one given by the pair-diagram term. The separation of the covariant result in such contributions is unique and does not depend on any particular dynamical model used to generate our choice of BS vertex, as long as the four-dimensional impulse approximation is adopted to calculate the em current (see Eq. (3) ).

Following Ref. [36], but with a nonconstant, symmetric vertex function, we have investigated the frame dependence of the pair-term contribution to the form factor of pionlike systems. Our conclusions strengthen and generalize the conclusions of Ref. [36]. In particular, after introducing a transverse momentum distribution to distinguish between strongly and weakly relativistic systems, we have also analyzed the form factor for the second case.

In general the magnitude of the pair-term contribution in the electromagnetic current depends on the frame, because a dynamical transformation change the relative weight of valence and nonvalence contributions in the Fock state and then in the form factor, while the sum, that is an invariant quantity, does not. For strongly relativistic systems, as the 
pion, the effect is dramatic: in the Breit frame where $q^{+}=\sqrt{-q^{2}}$ the form factor is largely dominated by the pair diagram for $-q^{2} \neq 0$, while adjusting the model to the deuteron scale (weakly relativistic system), the pair term becomes negligible at low momentum transfer and only contributes by $30 \%$ at $-q^{2}=4(\mathrm{GeV} / \mathrm{c})^{2}$.

The rapid fall-off of the valence component contribution to the form factor of the pion for purely longitudinal momentum transfers, $q^{+}=\sqrt{-q^{2}}$, is a feature which can be understood also by a general, crude kinematical estimate. As matter of fact, the ratio between the pair contribution and the full form factor is roughly given by (see also, e. g., [21])

$$
R^{K I N}=\frac{q^{+}}{\sqrt{m_{\pi}^{2}+\left(\frac{q^{+}}{2}\right)^{2}}+\frac{q^{+}}{2}} .
$$

From such an estimate one can have some insight on the role played by the mass of the composite system, in determining the onset of the importance of the pair diagram in the form factor.

Finally, we have compared the results of our covariant model for the pion form factor to the ones developed within the Front Form Hamiltonian Dynamics, based on one-body current operators. Such a comparison has shown that some general features are shared. In particular, the fall-off of the valence contribution in the $q^{+}=\sqrt{-q^{2}}$ frame faster than the one in the $q^{+}=0$ frame can be recovered in the FFHD approach. It should be pointed out that the FFHD calculations do not include the contribution of the pair term to the form factor, which should appear as a two-body current contribution.

In the case of the deuteron scale (weakly relativistic system), the relevance of the pairterm contribution is shifted towards higher values of the momentum transfer $\left(2-4(\mathrm{GeV} / \mathrm{c})^{2}\right)$. Therefore, these encouraging results of our exploratory analysis at the deuteron scale urge studies which take into account the vector nature of the deuteron.

The investigation of the pair-term contribution carried out within the covariant model of the present work could give a first suggestion for an explicit form of the two-body current contribution to be used within phenomenological FFHD approaches. A detailed analysis of the light-front pair term in a given field theoretical model can be done using the quasipotential approach of Refs. [5, 6]. Within this approach, the light-front pair diagram for nonvanishing $q^{+}$will come from the contribution of a two-body current, which can be formally derived in a consistent way. Numerical investigations of a dynamical model, featuring the mentioned properties and suitable for applications to hadrons, will be presented elsewhere.

\section{Acknowledgments}

We gratefully acknowledge Felix Lev for interesting discussions about the symmetry properties of the vertex function. This work was partially supported by the Brazilian agencies CNPq and FAPESP and by Ministero della Ricerca Scientifica e Tecnologica. T.F. acknowledges the hospitality of the Dipartimento di Fisica, Università di Roma "Tor Vergata" and of Istituto Nazionale di Fisica Nucleare, Sezione Tor Vergata and Sezione Roma I. 


\section{A Analytic Integration on $k^{-}$in $F_{\pi}^{(I)}$}

In this Appendix, we show in detail how to perform the contour integration on $k^{-}$in Eq. (7) for $F_{\pi}^{(I)}\left(q^{2}, \alpha\right)$. This quantity can be rewritten as follows

$$
F_{\pi}^{(I)}\left(q^{2}, \alpha\right)=F_{\pi}^{(I) a}+F_{\pi}^{(I) b}+F_{\pi}^{(I) c}+F_{\pi}^{(I) d}
$$

The first term in Eq. (36), $F_{\pi}^{(I) a}$, is

$$
\begin{aligned}
F_{\pi}^{(I) a} & =-\frac{\imath}{2 \pi} N \int \frac{d^{2} k_{\perp} d k^{+} d k^{-} \theta\left(k^{+}\right) \theta\left(P^{+}-k^{+}\right)}{\left(k^{+}\right)^{3}\left(P^{+}-k^{+}\right)\left(P^{\prime+}-k^{+}\right)} \operatorname{Tr}\left[\mathcal{O}^{+}\left(k^{-}\right)\right] \\
& \times \frac{1}{\left(k^{-}-k_{o n}^{-}+\imath \epsilon\right)\left(P^{-}-k^{-}-(P-k)_{o n}^{-}+\imath \epsilon\right)} \\
& \times \frac{1}{\left(P^{\prime-}-k^{-}-\left(P^{\prime}-k\right)_{o n}^{-}+\imath \epsilon\right)\left(k^{-}-k_{R}^{-}+\imath \epsilon\right)^{2}},
\end{aligned}
$$

where we explicitly wrote the dependence upon $k^{-}$in $\operatorname{Tr}\left[\mathcal{O}^{+}\left(k^{-}\right)\right]$and

$$
k_{R}^{-}=\frac{k_{\perp}^{2}+m_{R}^{2}}{k^{+}}
$$

The on-energy-shell values of the individual momenta $k_{o n}^{-},(P-k)_{\text {on }}^{-}$and $\left(P^{\prime}-k\right)_{\text {on }}^{-}$are given by Eq. (10). The normalization factor is

$$
N=\frac{m^{2} C^{2}}{\left(P^{+}+P^{\prime+}\right) f_{\pi}^{2}} \frac{N_{c}}{(2 \pi)^{3}}
$$

For the sake of algebraic simplicity the contour of integration in Eq. (37) can be

closed in the upper complex semi-plane of $k^{-}$, where only the poles $k_{(1)}^{-}$and $k_{(3)}^{-}$, given by Eqs. (14) and (16), respectively, are present. By evaluating the residues of the integrand in Eq. (37), one has

$$
\begin{aligned}
F_{\pi}^{(I) a} & =-N \int \frac{d^{2} k_{\perp} d k^{+} \theta\left(k^{+}\right) \theta\left(P^{+}-k^{+}\right)}{\left(k^{+}\right)^{3}\left(P^{+}-k^{+}\right)\left(P^{\prime}+k^{+}\right)} \frac{1}{\left(P^{\prime-}-P^{-}+(P-k)_{o n}^{-}-\left(P^{\prime}-k\right)_{o n}^{-}\right)} \\
& \times\left[\frac{\operatorname{Tr}\left[\mathcal{O}^{+}\left(P^{-}-(P-k)_{o n}^{-}\right)\right]}{\left(P^{-}-(P-k)_{o n}^{-}-k_{o n}^{-}\right)\left(P^{-}-(P-k)_{o n}^{-}-k_{R}^{-}\right)^{2}}\right. \\
& \left.-\frac{\operatorname{Tr}\left[\mathcal{O}^{+}\left(P^{\prime-}-\left(P^{\prime}-k\right)_{o n}^{-}\right)\right]}{\left(P^{\prime-}-\left(P^{\prime}-k\right)_{o n}^{-}-k_{o n}^{-}\right)\left(P^{\prime-}-\left(P^{\prime}-k\right)_{o n}^{-}-k_{R}^{-}\right)^{2}}\right] .
\end{aligned}
$$

The second term in Eq. (36), $F_{\pi}^{(I) b}$, is given by

$$
\begin{aligned}
F_{\pi}^{(I) b} & =-\frac{\imath}{2 \pi} N \int \frac{d^{2} k_{\perp} d k^{+} d k^{-} \theta\left(k^{+}\right) \theta\left(P^{+}-k^{+}\right)}{\left(k^{+}\right)^{2}\left(P^{+}-k^{+}\right)\left(P^{\prime+}-k^{+}\right)^{2}} \operatorname{Tr}\left[\mathcal{O}^{+}\left(k^{-}\right)\right] \\
& \times \frac{1}{\left(k^{-}-k_{o n}^{-}+\imath \epsilon\right)\left(P^{-}-k^{-}-(P-k)_{o n}^{-}+\imath \epsilon\right)\left(P^{\prime-}-k^{-}-\left(P^{\prime}-k\right)_{o n}^{-}+\imath \epsilon\right)} \\
& \times \frac{1}{\left(k^{-}-k_{R}^{-}+\imath \epsilon\right)\left(P^{\prime-}-k^{-}-\left(P^{\prime}-k\right)_{R}^{-}+\imath \epsilon\right)},
\end{aligned}
$$


where

$$
\left(P^{\prime}-k\right)_{R}^{-}=\frac{\left(P^{\prime}-k\right)_{\perp}^{2}+m_{R}^{2}}{P^{\prime+}-k^{+}} .
$$

In Eq. (41), the integration contour is closed in the lower complex semi-plane of $k^{-}$, where the poles $k_{(5)}^{-}=k_{o n}^{-}-\imath \epsilon$ and $k_{(6)}^{-}=k_{R}^{-}-\imath \epsilon$ are present. By calculating the residues of the integrand in Eq. (41), one gets

$$
\begin{aligned}
F_{\pi}^{(I) b} & =-N \int \frac{d^{2} k_{\perp} d k^{+} \theta\left(k^{+}\right) \theta\left(P^{+}-k^{+}\right)}{\left(k^{+}\right)^{2}\left(P^{+}-k^{+}\right)\left(P^{\prime+}-k^{+}\right)^{2}} \operatorname{Tr}\left[\mathcal{O}^{+}\left(k_{o n}^{-}\right)\right] \\
& \times \frac{1}{\left(P^{-}-(P-k)_{o n}^{-}-k_{o n}^{-}\right)\left(P^{\prime-}-\left(P^{\prime}-k\right)_{o n}^{-}-k_{o n}^{-}\right)} \\
& \times \frac{1}{\left(k_{o n}^{-}-k_{R}^{-}\right)\left(P^{\prime-}-k_{o n}^{-}-\left(P^{\prime}-k\right)_{R}^{-}\right)}+\left[k_{o n}^{-} \leftrightarrow k_{R}^{-}\right] .
\end{aligned}
$$

The third term in Eq. (36), $F_{\pi}^{(I) c}$, is given by

$$
\begin{aligned}
F_{\pi}^{(I) c} & =-\frac{\imath}{2 \pi} N \int \frac{d^{2} k_{\perp} d k^{+} d k^{-} \theta\left(k^{+}\right) \theta\left(P^{+}-k^{+}\right)}{\left(k^{+}\right)^{2}\left(P^{+}-k^{+}\right)\left(P^{\prime}-k^{+}\right)^{2}} \operatorname{Tr}\left[\mathcal{O}^{+}\left(k^{-}\right)\right] \\
& \times \frac{1}{\left(k^{-}-k_{o n}^{-}+\imath \epsilon\right)\left(P^{-}-k^{-}-(P-k)_{o n}^{-}+\imath \epsilon\right)\left(P^{\prime-}-k^{-}-\left(P^{\prime}-k\right)_{o n}^{-}+\imath \epsilon\right)} \\
& \times \frac{1}{\left(k^{-}-k_{R}^{-}+\imath \epsilon\right)\left(P^{-}-k^{-}-(P-k)_{R}^{-}+\imath \epsilon\right)} .
\end{aligned}
$$

Eq. (44) is identical to Eq. (41) with $P^{\prime} \leftrightarrow P$. Consequently we can write

$$
F_{\pi}^{(I) c}=F_{\pi}^{(I) b}\left[P^{\prime} \leftrightarrow P\right] .
$$

The last term in Eq. (36), $F_{\pi}^{(I) d}$, that represents the full $F_{\pi}^{(I)}$ for the nonsymmetric model of Ref. [22], is given by

$$
\begin{aligned}
F_{\pi}^{(I) d} & =-\frac{\imath}{2 \pi} N \int \frac{d^{2} k_{\perp} d k^{+} d k^{-} \theta\left(k^{+}\right) \theta\left(P^{+}-k^{+}\right)}{k^{+}\left(P^{+}-k^{+}\right)^{2}\left(P^{\prime+}-k^{+}\right)^{2}} \operatorname{Tr}\left[\mathcal{O}^{+}\left(k^{-}\right)\right] \\
& \times \frac{1}{\left(k^{-}-k_{o n}^{-}+\imath \epsilon\right)\left(P^{-}-k^{-}-(P-k)_{o n}^{-}+\imath \epsilon\right)\left(P^{\prime-}-k^{-}-\left(P^{\prime}-k\right)_{o n}^{-}+\imath \epsilon\right)} \\
& \times \frac{1}{\left(P^{-}-k^{-}-(P-k)_{R}^{-}+\imath \epsilon\right)\left(P^{\prime-}-k^{-}-\left(P^{\prime}-k\right)_{R}^{-}+\imath \epsilon\right)},
\end{aligned}
$$

where

$$
(P-k)_{R}^{-}=\frac{(P-k)_{\perp}^{2}+m_{R}^{2}}{P^{+}-k^{+}} .
$$


In Eq. (46), the integration contour can be closed in the lower complex semi-plane of $k^{-}$, where only the pole $k_{(5)}^{-}=k_{o n}^{-}-\imath \epsilon$ is present. By evaluating the residue of the integrand in Eq. (46), one has

$$
\begin{aligned}
F_{\pi}^{(I) d} & =-N \int \frac{d^{2} k_{\perp} d k^{+} \theta\left(k^{+}\right) \theta\left(P^{+}-k^{+}\right)}{k^{+}\left(P^{+}-k^{+}\right)^{2}\left(P^{\prime+}-k^{+}\right)^{2}} \operatorname{Tr}\left[\mathcal{O}^{+}\left(k_{o n}^{-}\right)\right] \\
& \times \frac{1}{\left(P^{-}-k_{o n}^{-}-(P-k)_{o n}^{-}\right)\left(P^{\prime-}-k_{o n}^{-}-\left(P^{\prime}-k\right)_{o n}^{-}\right)} \\
& \times \frac{1}{\left(P^{-}-k_{o n}^{-}-(P-k)_{R}^{-}\right)\left(P^{\prime-}-k_{o n}^{-}-\left(P^{\prime}-k\right)_{R}^{-}\right)} .
\end{aligned}
$$




\section{B Analytic Integration on $k^{-}$in $F_{\pi}^{(I I)}$}

To perform the contour integration on $k^{-}$in Eq. (8) for the pair term, $F_{\pi}^{(I I)}\left(q^{2}, \alpha\right)$, we rewrite such a contribution as follows

$$
F_{\pi}^{(I I)}\left(q^{2}, \alpha\right)=F_{\pi}^{(I I) a}+F_{\pi}^{(I I) b}+F_{\pi}^{(I I) c}+F_{\pi}^{(I I) d} .
$$

All the terms in Eq. (49) can be evaluated by closing the integration contour in the upper complex semi-plane of $k^{-}$. The first term in Eq. (49), $F_{\pi}^{(I I) a}$ is

$$
\begin{aligned}
F_{\pi}^{(I I) a} & =-\frac{\imath}{2 \pi} N \int \frac{d^{2} k_{\perp} d k^{+} d k^{-} \theta\left(k^{+}-P^{+}\right) \theta\left(P^{\prime+}-k^{+}\right)}{\left(k^{+}\right)^{3}\left(P^{+}-k^{+}\right)\left(P^{\prime+}-k^{+}\right)} \operatorname{Tr}\left[\mathcal{O}^{+}\left(k^{-}\right)\right] \\
& \times \frac{1}{\left(k^{-}-k_{o n}^{-}+\imath \epsilon\right)\left(P^{-}-k^{-}-(P-k)_{o n}^{-}-\imath \epsilon\right)} \\
& \times \frac{1}{\left(P^{\prime-}-k^{-}-\left(P^{\prime}-k\right)_{o n}^{-}+\imath \epsilon\right)\left(k^{-}-k_{R}^{-}+\imath \epsilon\right)^{2}}
\end{aligned}
$$

where the on-energy-shell values of the individual momenta $k_{\text {on }}^{-},(P-k)_{\text {on }}^{-}$and $\left(P^{\prime}-k\right)_{\text {on }}^{-}$ are again given by Eq. (10) and $k_{R}^{-}$is defined by Eq. (38). The normalization factor is the same as in Eq. (39).

In Eq. (50), only the pole $k_{(3)}^{-}$given by Eq. (16) is present in the upper complex semi-plane of $k^{-}$. By evaluating the residue of the integrand in Eq. (49), one gets

$$
\begin{aligned}
F_{\pi}^{(I I) a} & =-N \int \frac{d^{2} k_{\perp} d k^{+} \theta\left(k^{+}-P^{+}\right) \theta\left(P^{\prime+}-k^{+}\right)}{\left(k^{+}\right)^{3}\left(P^{+}-k^{+}\right)\left(P^{\prime}+k^{+}\right)} \frac{\operatorname{Tr}\left[\mathcal{O}^{+}\left(P^{\prime-}-\left(P^{\prime}-k\right)_{o n}^{-}\right)\right]}{\left(P^{\prime-}-\left(P^{\prime}-k\right)_{o n}^{-}-k_{o n}^{-}\right)} \\
& \times \frac{1}{\left(P^{-}-P^{\prime-}-(P-k)_{o n}^{-}+\left(P^{\prime}-k\right)_{o n}^{-}\right)\left(P^{\prime-}-\left(P^{\prime}-k\right)_{o n}^{-}-k_{R}^{-}\right)^{2}} .
\end{aligned}
$$

The second term in Eq. (49), $F_{\pi}^{(I I) b}$, is given by

$$
\begin{aligned}
F_{\pi}^{(I I) b} & =-\frac{\imath}{2 \pi} N \int \frac{d^{2} k_{\perp} d k^{+} d k^{-} \theta\left(k^{+}-P^{+}\right) \theta\left(P^{\prime+}-k^{+}\right)}{\left(k^{+}\right)^{2}\left(P^{+}-k^{+}\right)\left(P^{\prime+}-k^{+}\right)^{2}} \operatorname{Tr}\left[\mathcal{O}^{+}\left(k^{-}\right)\right] \\
& \times \frac{1}{\left(k^{-}-k_{o n}^{-}+\imath \epsilon\right)\left(P^{-}-k^{-}-(P-k)_{o n}^{-}-\imath \epsilon\right)\left(P^{\prime-}-k^{-}-\left(P^{\prime}-k\right)_{o n}^{-}+\imath \epsilon\right)} \\
& \times \frac{1}{\left(k^{-}-k_{R}^{-}+\imath \epsilon\right)\left(P^{\prime-}-k^{-}-\left(P^{\prime}-k\right)_{R}^{-}+\imath \epsilon\right)} .
\end{aligned}
$$

In Eq. (52), the poles $k_{(3)}^{-}$, Eq. (16), and $k_{(4)}^{-}$, Eq. (17), contribute. By calculating the residues of the integrand in Eq. (52), one has

$$
\begin{aligned}
F_{\pi}^{(I I) b} & =-N \int \frac{d^{2} k_{\perp} d k^{+} \theta\left(k^{+}-P^{+}\right) \theta\left(P^{\prime+}-k^{+}\right)}{\left(k^{+}\right)^{2}\left(P^{+}-k^{+}\right)\left(P^{\prime+}-k^{+}\right)^{2}} \operatorname{Tr}\left[\mathcal{O}^{+}\left(P^{\prime-}-\left(P^{\prime}-k\right)_{\text {on }}^{-}\right)\right] \\
& \times \frac{1}{\left(\left(P^{\prime}-k\right)_{o n}^{-}-\left(P^{\prime}-k\right)_{R}^{-}\right)\left(P^{\prime-}-\left(P^{\prime}-k\right)_{o n}^{-}-k_{o n}^{-}\right)}
\end{aligned}
$$




$$
\begin{aligned}
& \times \frac{1}{\left(P^{\prime-}-\left(P^{\prime}-k\right)_{o n}^{-}-k_{R}^{-}\right)\left(P^{-}-P^{\prime-}+\left(P^{\prime}-k\right)_{o n}^{-}-(P-k)_{o n}^{-}\right)} \\
& +\left[\left(P^{\prime}-k\right)_{o n}^{-} \leftrightarrow\left(P^{\prime}-k\right)_{R}^{-}\right] .
\end{aligned}
$$

The third term in Eq. (49), $F_{\pi}^{(I I) c}$, is

$$
\begin{aligned}
F_{\pi}^{(I I) c} & =-\frac{\imath}{2 \pi} N \int \frac{d^{2} k_{\perp} d k^{+} d k^{-} \theta\left(k^{+}-P^{+}\right) \theta\left(P^{\prime+}-k^{+}\right)}{\left(k^{+}\right)^{2}\left(P^{+}-k^{+}\right)\left(P^{\prime}+k^{+}\right)^{2}} \operatorname{Tr}\left[\mathcal{O}^{+}\left(k^{-}\right)\right] \\
& \times \frac{1}{\left(k^{-}-k_{o n}^{-}+\imath \epsilon\right)\left(P^{-}-k^{-}-(P-k)_{o n}^{-}-\imath \epsilon\right)\left(P^{\prime-}-k^{-}-\left(P^{\prime}-k\right)_{o n}^{-}+\imath \epsilon\right)} \\
& \times \frac{1}{\left(k^{-}-k_{R}^{-}+\imath \epsilon\right)\left(P^{-}-k^{-}-(P-k)_{R}^{-}-\imath \epsilon\right)} .
\end{aligned}
$$

In Eq. (54) the only pole of the integrand in the upper complex semi-plane of $k^{-}$is $k_{(3)}^{-}$, given by Eq. (16). By computing the residue, the result is

$$
\begin{aligned}
F_{\pi}^{(I I) c} & =-N \int \frac{d^{2} k_{\perp} d k^{+} \theta\left(k^{+}-P^{+}\right) \theta\left(P^{\prime+}-k^{+}\right)}{\left(k^{+}\right)^{2}\left(P^{+}-k^{+}\right)\left(P^{\prime+}-k^{+}\right)^{2}} \operatorname{Tr}\left[\mathcal{O}^{+}\left(P^{\prime-}-\left(P^{\prime}-k\right)_{o n}^{-}\right)\right] \\
& \times \frac{1}{\left(P^{\prime-}-\left(P^{\prime}-k\right)_{o n}^{-}-k_{o n}^{-}\right)\left(P^{-}-P^{\prime-}+\left(P^{\prime}-k\right)_{o n}^{-}-(P-k)_{o n}^{-}\right)} \\
& \times \frac{1}{\left(P^{\prime-}-\left(P^{\prime}-k\right)_{o n}^{-}-k_{R}^{-}\right)\left(P^{-}-P^{\prime-}+\left(P^{\prime}-k\right)_{o n}^{-}-(P-k)_{R}^{-}\right)} .
\end{aligned}
$$

The last term is Eq. (49), $F_{\pi}^{(I I) d}$, is given by

$$
\begin{aligned}
F_{\pi}^{(I I) d} & =-\frac{\imath}{2 \pi} N \int \frac{d^{2} k_{\perp} d k^{+} d k^{-} \theta\left(k^{+}-P^{+}\right) \theta\left(P^{\prime+}-k^{+}\right)}{k^{+}\left(P^{+}-k^{+}\right)^{2}\left(P^{\prime+}-k^{+}\right)^{2}} \operatorname{Tr}\left[\mathcal{O}^{+}\left(k^{-}\right)\right] \\
& \times \frac{1}{\left(k^{-}-k_{o n}^{-}+\imath \epsilon\right)\left(P^{-}-k^{-}-(P-k)_{o n}^{-}-\imath \epsilon\right)\left(P^{\prime-}-k^{-}-\left(P^{\prime}-k\right)_{o n}^{-}+\imath \epsilon\right)} \\
& \times \frac{1}{\left(P^{-}-k^{-}-(P-k)_{R}^{-}-\imath \epsilon\right)\left(P^{\prime-}-k^{-}-\left(P^{\prime}-k\right)_{R}^{-}+\imath \epsilon\right)}
\end{aligned}
$$

In Eq. (56) the poles $k_{(3)}^{-}$, Eq. (16), and $k_{(4)}^{-}$, Eq. (17), contribute. By calculating the residues of the integrand of Eq. (56), we obtain

$$
\begin{aligned}
F_{\pi}^{(I I) d} & =-N \int \frac{d^{2} k_{\perp} d k^{+} \theta\left(k^{+}-P^{+}\right) \theta\left(P^{\prime+}-k^{+}\right)}{k^{+}\left(P^{+}-k^{+}\right)^{2}\left(P^{\prime}+k^{+}\right)^{2}} \operatorname{Tr}\left[\mathcal{O}^{+}\left(P^{\prime-}-\left(P^{\prime}-k\right)_{\text {on }}^{-}\right)\right] \\
& \times \frac{1}{\left(P^{\prime-}-\left(P^{\prime}-k\right)_{\text {on }}^{-}-k_{\text {on }}^{-}\right)\left(P^{-}-P^{\prime-}+\left(P^{\prime}-k\right)_{\text {on }}^{-}-(P-k)_{\text {on }}^{-}\right)} \\
& \times \frac{1}{\left(P^{-}-P^{\prime-}+\left(P^{\prime}-k\right)_{\text {on }}^{-}-(P-k)_{R}^{-}\right)\left(\left(P^{\prime}-k\right)_{\text {on }}^{-}-\left(P^{\prime}-k\right)_{R}^{-}\right)} \\
& +\left[\left(P^{\prime}-k\right)_{\text {on }}^{-} \leftrightarrow\left(P^{\prime}-k\right)_{R}^{-}\right] .
\end{aligned}
$$




\section{References}

[1] P. A. M. Dirac, Rev. Mod. Phys. 21, 392 (1949).

[2] S. J. Brodsky, H. C. Pauli, and S. S. Pinsky, Phys. Rep. 301, 299 (1998).

[3] H. C. Pauli, Eur. Phys. J. C7, (1998) 289.

[4] H. C. Pauli, "DLCQ and the effective interactions in hadrons" in: New Directions in Quantum Chromodynamics, C.R. Ji and D.P. Min, Editors, American Institute of Physics, 1999, p. 80-139.

[5] J. H. O. Sales, T. Frederico, B. V. Carlson and P. U. Sauer, Phys. Rev. C61, 044003 (2000).

[6] J. H. O. Sales, T. Frederico, B. V. Carlson and P. U. Sauer, Phys. Rev. C63, 064003 (2001).

[7] G. A. Miller, Progr. Part. Nucl. Phys. 45, 83 (2000); J. R. Cooke, G. A. Miller and D. R. Phillips, Phys. Rev. C61, 064005 (2000); J. R. Cooke and G. A. Miller, Phys. Rev.C62, 054008 (2000).

[8] M. Mangin-Brinet and J. Carbonell, Phys. Lett. B474, 237 (2000); M. Mangin-Brinet, J. Carbonell and V. A. Karmanov, Nucl. Phys. B Proc. Suppl. 90, 103 (2000); Phys. Rev. D64 , 027701 (2001); V. A. Karmanov, M. Mangin-Brinet and J. Carbonell, Nucl. Phys. A684, 366 (2001).

[9] B. Bakamjian and L. H. Thomas, Phys. Rev. 92, 1300 (1953).

[10] B. D. Keister and W. N. Polyzou, Adv. Nucl. Phys. 20, 225 (1991).

[11] F.M. Lev, Rivista Nuovo Cimento 16, 1 (1993).

[12] P. L. Chung, F. Coester, B. D. Keister, and W. N. Polyzou, Phys. Rev. C37, 2000 (1988); P. L. Chung, B. D. Keister, F. Coester, ibid. 39, 1544 (1989).

[13] P. L. Chung, F. Coester and W. N. Polyzou, Phys. Lett. B205, 545 (1988); P. L. Chung and F. Coester, Phys. Rev. D44, 229 (1991).

[14] H. J. Weber, Ann. Phys. 177, 38 (1987); W. Konen and H. J. Weber, Phys. Rev. D41, 2201 (1990).

[15] S. Capstick and B. D. Keister Phys.Rev. D51,3598 (1995).

[16] F. Cardarelli, E. Pace, G. Salmè and S. Simula, Phys. Lett. B357, 267 (1995); Phys. Lett. B371, 7 (1996); Phys. Lett. B397, 13 (1997); Nucl. Phys. A666 \& A667, 33c (2000). 
[17] F. Cardarelli et al., Phys. Rev. D53, 6682 (1996).

[18] W. Jaus, Phys. Rev. D60, 054026 (1999); ibid. D63, 053009 (2001).

[19] F. M. Lev, E. Pace and G. Salmè, Phys. Rev. Lett. 83, 5250 (1999).

[20] L.L. Frankfurt and M.I. Strikman, Nucl. Phys. B148, 107 (1979).

[21] M. Sawicki, Phys. Rev. D44, 433(1991); Phys. Rev. D46, 474 (1992).

[22] J. P. B. C. de Melo, H. W. Naus and T. Frederico, Phys. Rev. C59, 2278 (1999).

[23] J. P. B. C. de Melo, J. H. O. Sales, T. Frederico and P. U. Sauer, Nucl. Phys. A631, 574c (1998).

[24] J. P. B. C. de Melo, T. Frederico, H. W. L. Naus and P. U. Sauer, Nucl.Phys. A660, 219 (1999).

[25] H. W. L. Naus, J. P. B. C. de Melo and T. Frederico, Few-Body Syst. 24, 99 (1998).

[26] I. L. Grach and L. A. Kondratyuk, Sov. J. Nucl. Phys. 39, 198 (1984).

[27] T. Frederico, E. M. Henley and G. A. Miller, Nucl. Phys. A533, 617 (1991).

[28] B. D. Keister, Phys. Rev. D49, 1500 (1994).

[29] F. Cardarelli, I.L. Grach, I.M. Narodetskii, G. Salmè and S. Simula, Phys. Lett. B359, 1 (1995).

[30] J. P. B. C. de Melo and T. Frederico, Phys. Rev. C55, 2043 (1997).

[31] L. L. Frankfurt, T. Frederico and M. I. Strikman, Phys. Rev. C48, 2182 (1993).

[32] J. Carbonell, B. Deplanques, V. A. Karmanov and J.-F. Mathiot, Phys. Rep. 300, 215 (1998).

[33] F. M. Lev, E. Pace and G. Salmè, Nucl. Phys. A641, 229 (1998); Few-Body Syst. Suppl. 10, 135 (1998).

[34] F. M. Lev, E. Pace and G. Salmè, Phys. Rev. C62, 064004 (2000); Nucl. Phys. A663, $365(2000)$.

[35] E. Pace and G. Salmè, Nucl. Phys. A684, 487 (2001); A689, 411 (2001).

[36] B. L. G. Bakker, H.-M. Choi and C.-R. Ji, Phys. Rev. D63, 074014 (2001).

[37] S. Godfrey and N. Isgur, Phys. Rev. D32, 185 (1985). 
[38] T. Frederico and G. A. Miller, Phys. Rev. D45, 4207 (1992); Phys. Rev. D50, 210 (1994).

[39] S.J. Brodsky and J. R. Primack, Ann. Phys. 52, 315 (1969), and references therein quoted.

[40] F. Coester and D.O. Riska, Ann. Phys. 234, 141 (1994).

[41] H.-M. Choi and C.-R. Ji, Phys. Rev. D58, 071901 (1998); Phys. Rev. D59, 034001 (1999).

[42] F. Cardarelli, I.L. Grach, I.M. Narodetskii, E. Pace, G. Salmè and S. Simula, Phys. Lett. B332, 1 (1994).

[43] S. R. Amendolia et al., Phys. Lett. B178, 116 (1986).

[44] J. Volmer et al., Phys. Rev. Lett. 86, 1713 (2001).

[45] C. N. Brown et al., Phys. Rev. D8, 92 (1973).

[46] C. J. Bebek et al., Phys. Rev. D9, 1229 (1974).

[47] C. J. Bebek et al., Phys. Rev. D13, 25 (1976).

[48] C. J. Bebek et al., Phys. Rev. D17, 1693 (1978).

[49] R. V. Reid, Ann. Phys. (N.Y.) 50, 411 (1968). 


\section{FIGURE CAPTIONS}

Fig. 1. Light-front time-ordered diagrams for the current: (a) $F_{\pi}^{(I)}$ (Eq. (7)) and (b) $F_{\pi}^{(I I)}(\mathrm{Eq} .(8))$.

Fig. 2. The pion form factor vs $-q^{2}$. The contributions to $F_{\pi}\left(q^{2}\right)$, evaluated in the Breit frame with $q^{+}=\sqrt{-q^{2}}$, i.e. $\alpha=90^{\circ}$ (Eq. (四), are also shown. Solid line: full result; dashed line: $F_{\pi}^{(I)}\left(q^{2}, \alpha\right)$ (full result without the pair term, Eq. (7) ); dotted line: $F_{\pi}^{(I I)}\left(q^{2}, \alpha\right)$ (pair term, Eq. (8)); long-dashed line: $F_{\pi}^{(I)}{ }_{\text {inst }}\left(q^{2}, \alpha\right)$ ( instantaneous-term contribution to $\left.F_{\pi}^{(I)}\left(q^{2}, \alpha\right)\right)$; short-dashed line: $F_{\pi}^{(I I)}$ inst $\left(q^{2}, \alpha\right)$ ( instantaneous-term contribution to the pair term). Experimental data: Ref. [44] (full squares), Ref. [45] (full triangles), Ref. [46] (empty squares), Ref. 47] (empty circles) and Ref. 448 (full circles).

Fig. 3. Contributions to the pion form factor vs $\alpha$ (see Eq. (4i) for $-q^{2}=1(\mathrm{GeV} / \mathrm{c})^{2}$. Solid line: full result; dashed line: $F_{\pi}^{(I)}\left(q^{2}, \alpha\right)$ (full result without the pair term, Eq. (đ))); dotted line: $F_{\pi}^{(I I)}\left(q^{2}, \alpha\right)$ (pair term, Eq. (8)); long-dashed line: $F_{\pi}^{(I)}$ inst $\left(q^{2}, \alpha\right)$ ( instantaneousterm contribution to $\left.F_{\pi}^{(I)}\left(q^{2}, \alpha\right)\right)$; short-dashed line: $F_{\pi}^{(I I)}$ inst $\left(q^{2}, \alpha\right)$ (instantaneous-term contribution to the pair term).

Fig. 4. Comparison between the pion form factor calculations for $F_{\pi}^{(I)}$ (Eq. (7)) and for $F_{\pi}^{(W F)}$ (Eq. (23)), at $\alpha=0^{\circ}, 45^{\circ}$ and $90^{\circ}$. Solid line: $F_{\pi}^{(I)}$; dotted line: $F_{\pi}^{(W F)}$, normalized to 1 at $q^{2}=0$.

Fig. 5. Form factor for a weakly relativistic spin-0 system vs $-q^{2}$. The contributions to the form factor, evaluated in the Breit frame with $q^{+}=\sqrt{-q^{2}}$, i.e. $\alpha=90^{\circ}$ (Eq. (4) ), are also shown. Solid line: full result; dashed line: $F_{\pi}^{(I)}\left(q^{2}, \alpha\right)$ (full result without the pair term, Eq. (7)); dotted line: $F_{\pi}^{(I I)}\left(q^{2}, \alpha\right)$ (pair term, Eq. (8)); long-dashed line: $\left|F_{\pi \text { inst }}^{(I)}\left(q^{2}, \alpha\right)\right|$ ( instantaneous-term contribution to $F_{\pi}^{(I)}\left(q^{2}, \alpha\right)$ ); short-dashed line: $F_{\pi \text { inst }}^{(I I)}\left(q^{2}, \alpha\right)$ (instantaneous-term contribution to the pair term).

Fig. 6. Dimensionless product of the transverse momentum probability density (Eq. (24)) times $k_{\perp}^{2}$ vs the ratio $k_{\perp} / m$. Results for the present pion model (solid curve with dots), the pion model of Ref.[15] (dashed curve), a weakly relativistic system (solid curve) and the deuteron from the Reid soft-core potential 49] (short-dashed curve).

Fig. 7. Ratio between the pair term, calculated for $\alpha=90^{\circ}$, and the full form factor vs $-q^{2}$. Upper curves correspond to the pion. Solid line: covariant pion model, $R^{(\mathrm{COV})}$ (Eq. (33); dashed line with dots: FFHD ratio, $R^{(F F H D)}$ (Eq. (35)); short-dashed line: kinematical estimate, $R^{(K I N)}$ (Eq. (34)). Lower curves correspond to a weakly relativistic system at the deuteron scale. Solid line: covariant result; dashed line: kinematical estimate. 


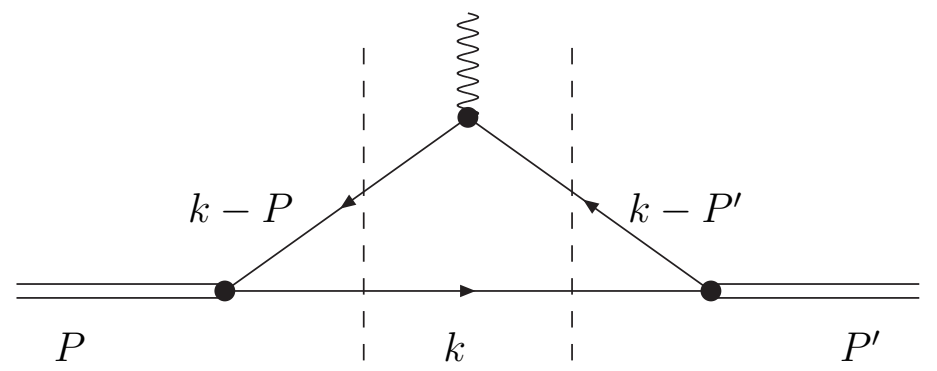

(a)

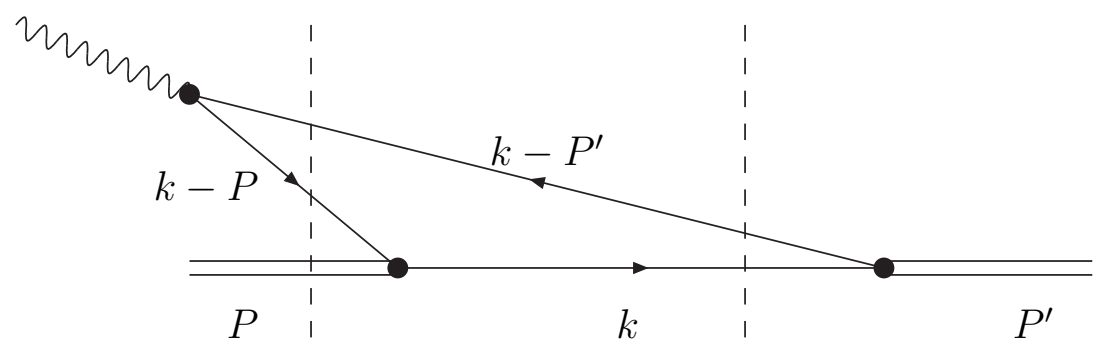

(b)

Fig. 1 - J. P. B. C. de Melo, T. Frederico, E. Pace and G.Salmè 


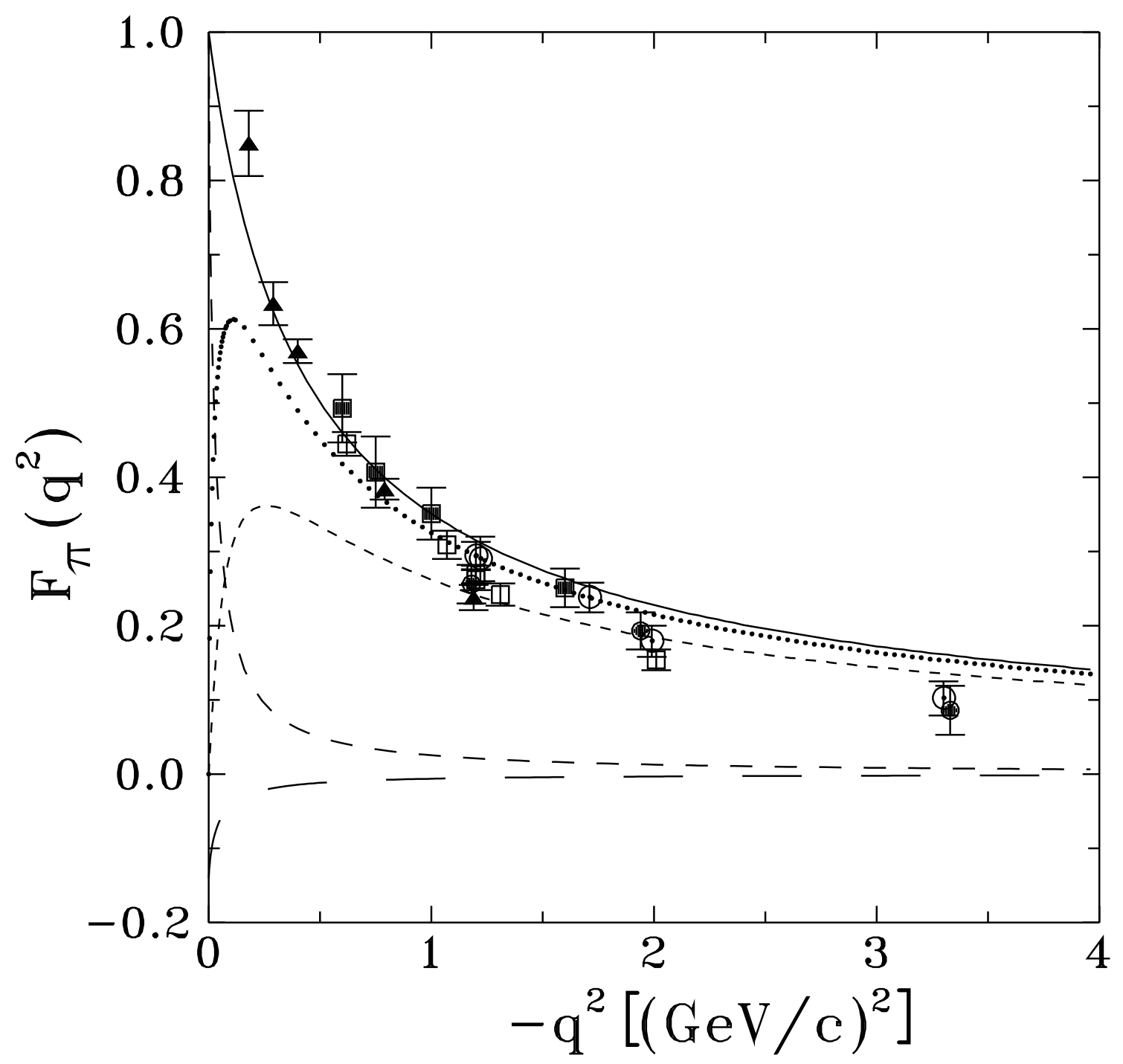

Fig. 2 - J. P. B. C. de Melo, T. Frederico, E. Pace and G. Salmè 


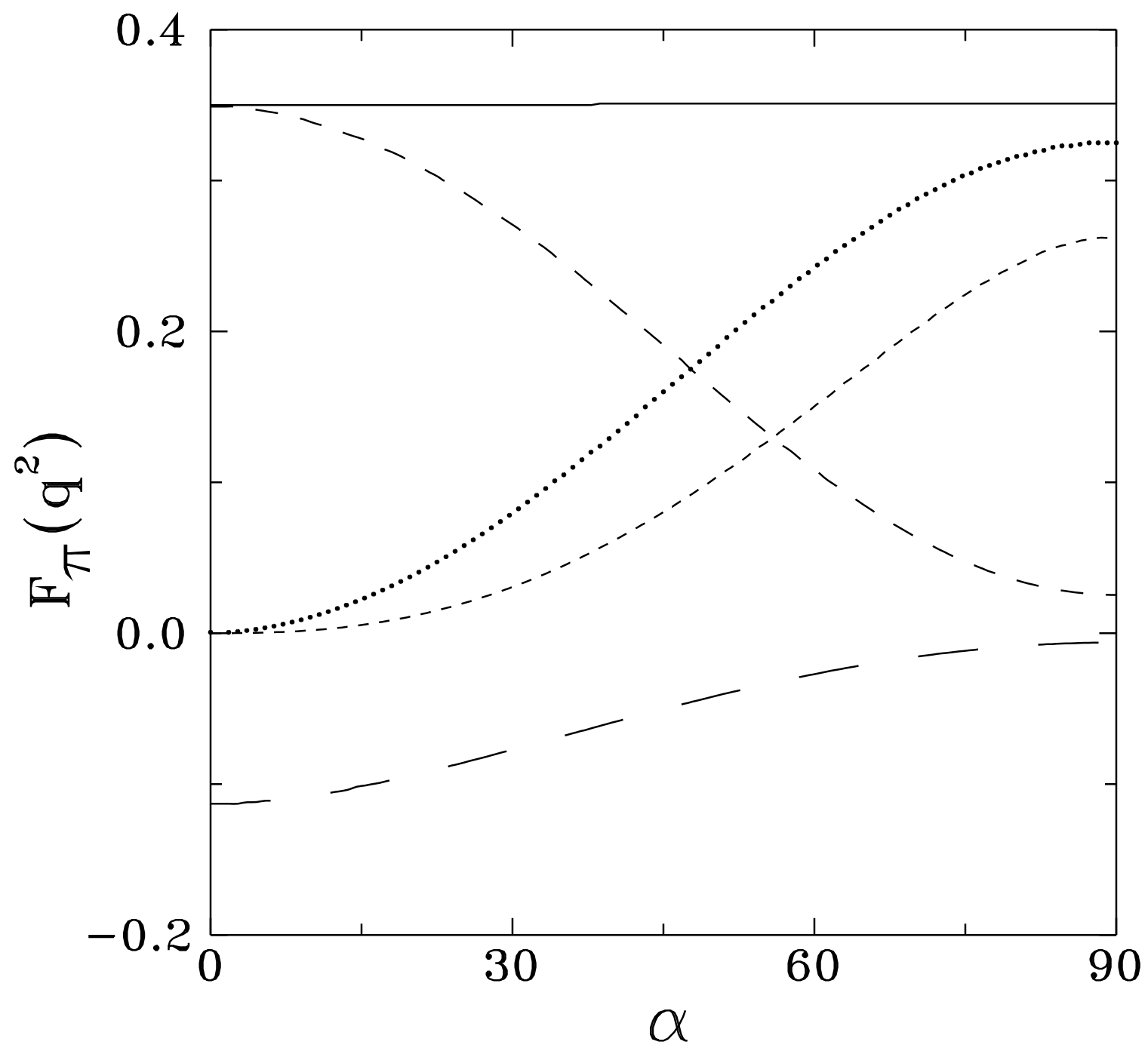

Fig. 3 - J. P. B. C. de Melo, T. Frederico, E. Pace and G. Salmè 


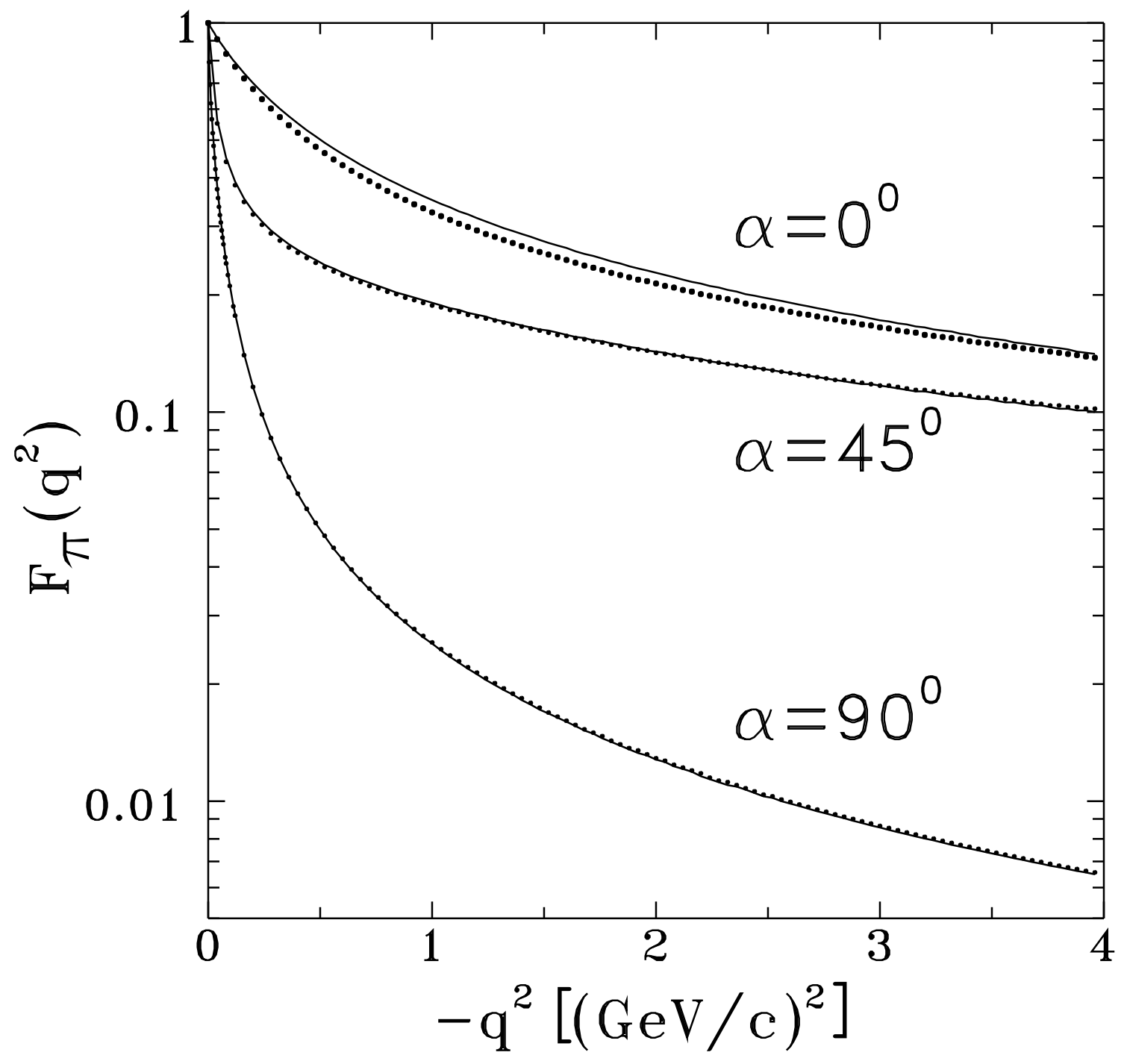

Fig. 4 - J. P. B. C. de Melo, T. Frederico, E. Pace and G. Salmè 


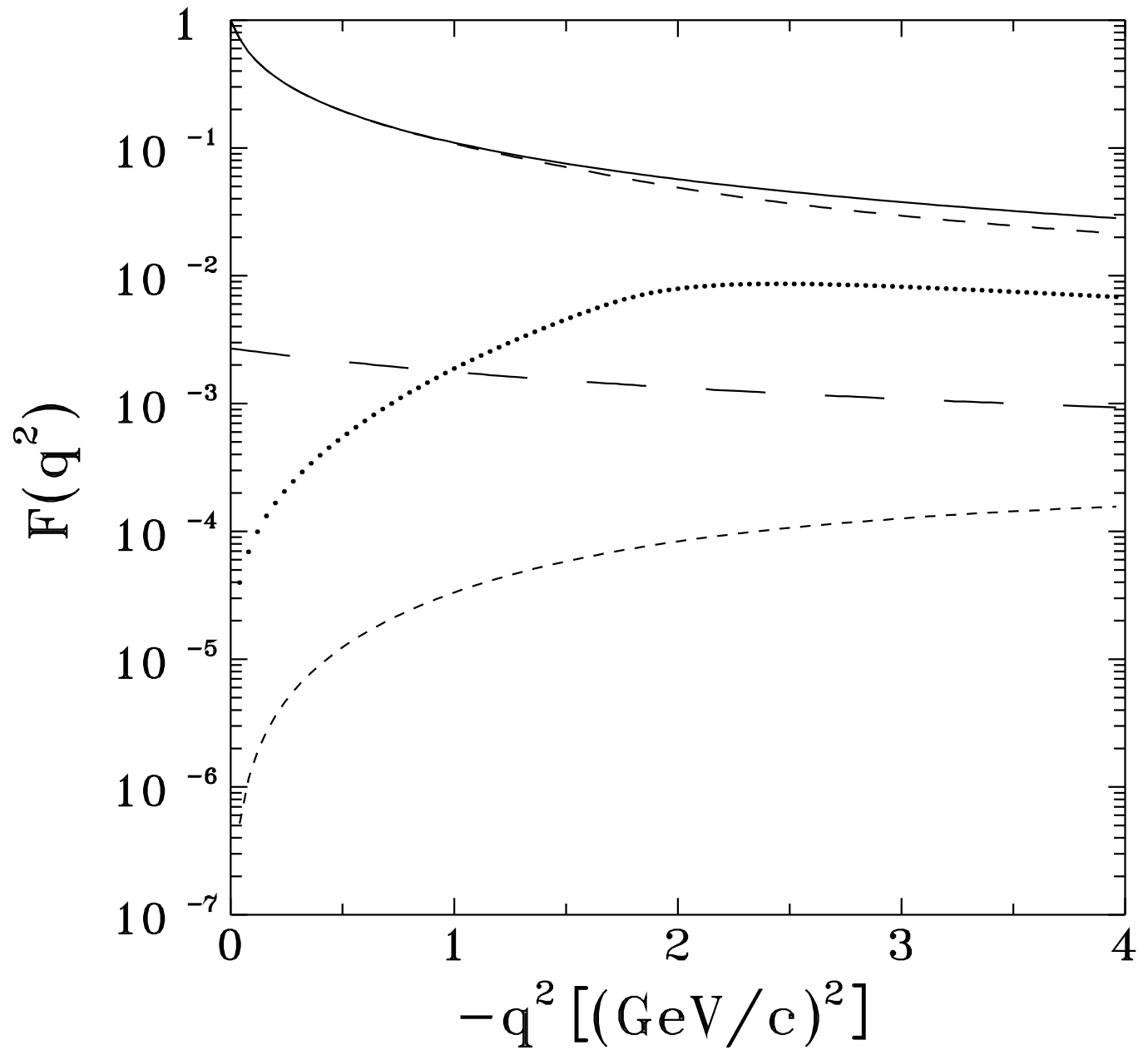

Fig. 5 - J. P. B. C. de Melo, T. Frederico, E. Pace and G. Salmè 


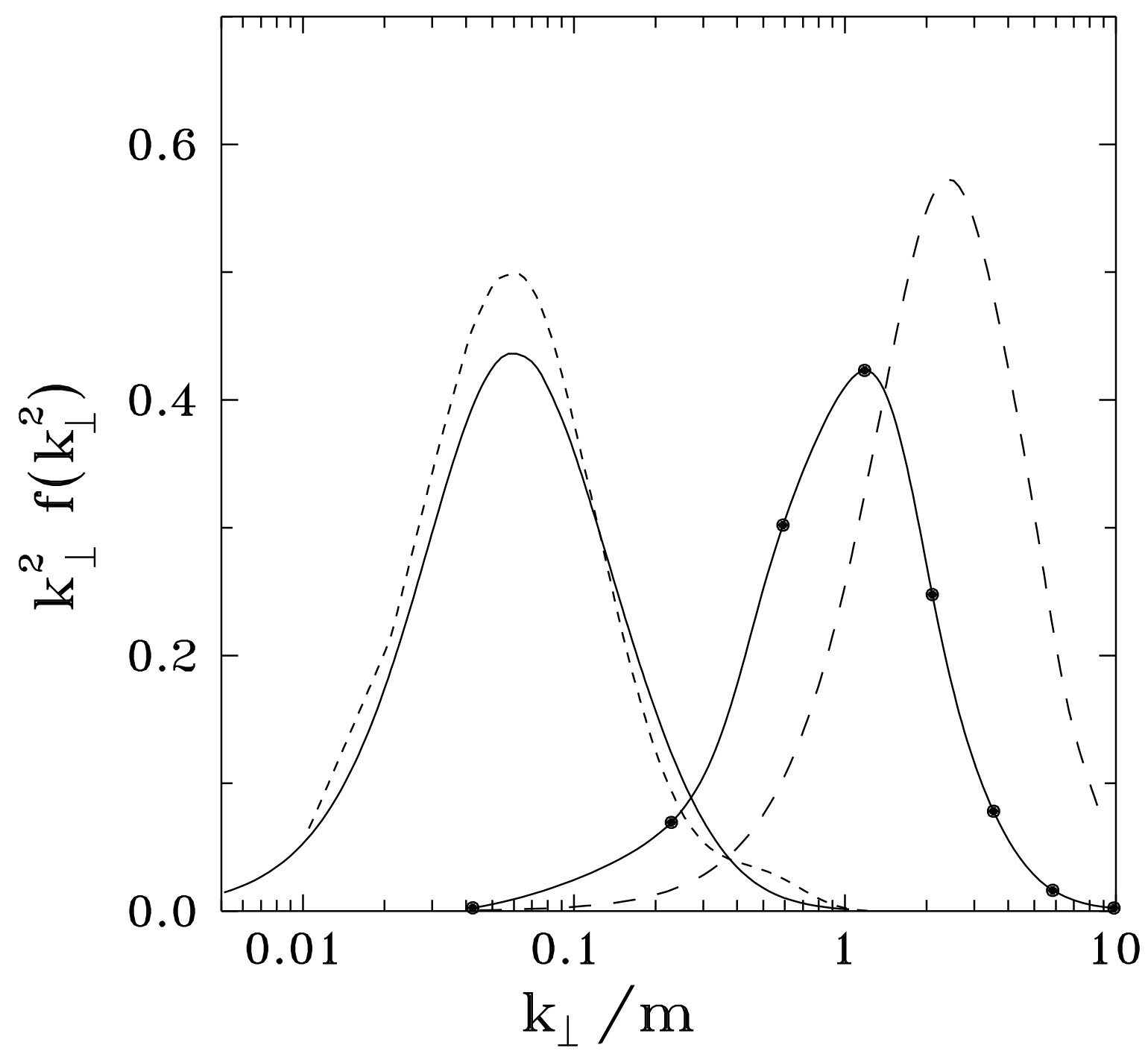

Fig. 6 - J. P. B. C. de Melo, T. Frederico, E. Pace and G. Salmè 


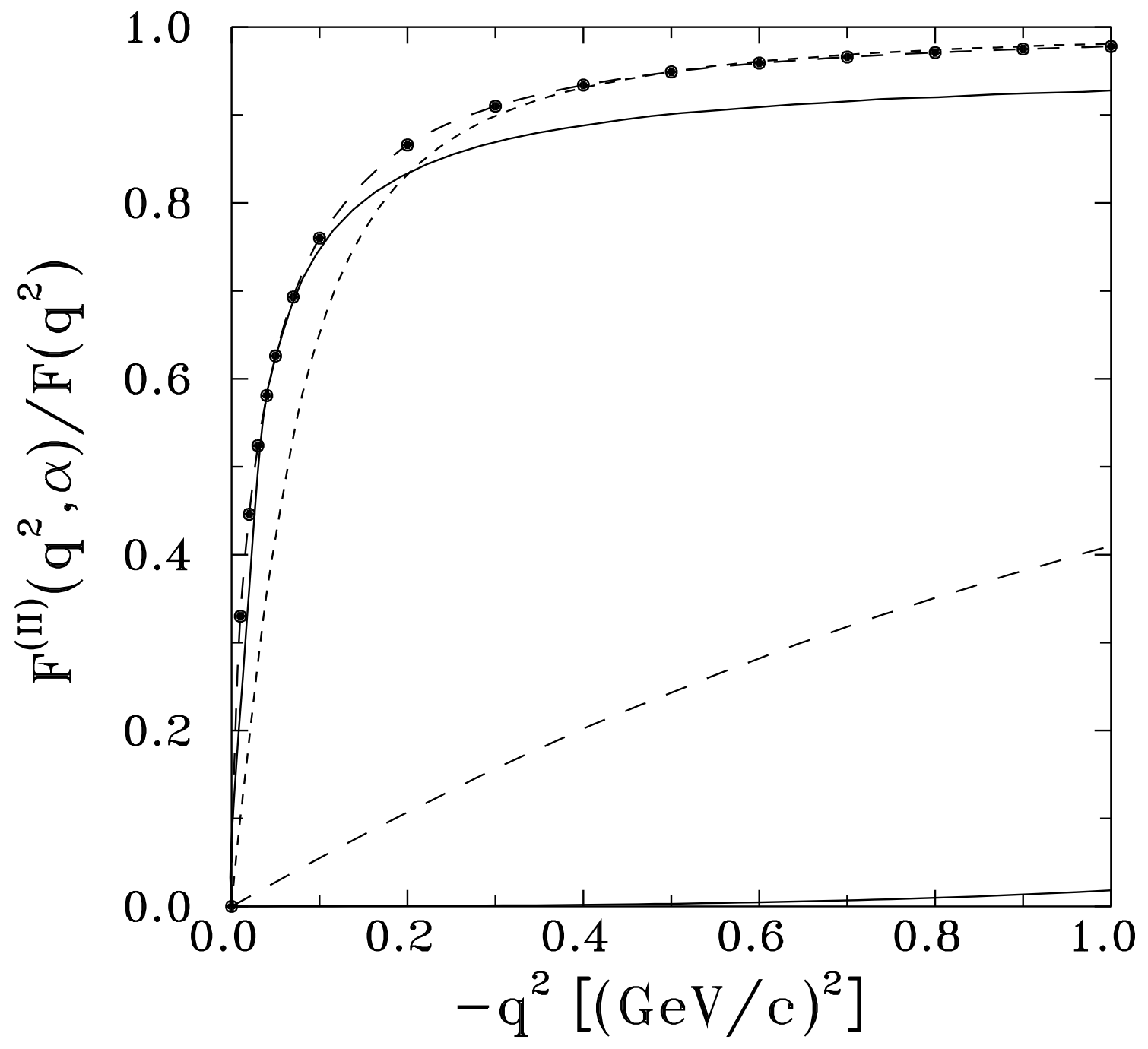

Fig. 7 - J. P. B. C. de Melo, T. Frederico, E. Pace and G. Salmè 